\title{
Subspace structure of some operator and Banach spaces
}

\author{
TIMUR OIKHBERG \\ CHRISTIAN ROSENDAL
}

\begin{abstract}
We construct a family of separable Hilbertian operator spaces, such that the relation of complete isomorphism between the subspaces of each member of this family is complete $\mathbf{K}_{\sigma}$. We also investigate some interesting properties of completely unconditional bases of the spaces from this family. In the Banach space setting, we construct a space for which the relation of isometry of subspaces is equivalent to equality of real numbers.
\end{abstract}

2000 Mathematics Subject Classification 46L07 (primary); 03E15 (secondary)

Keywords: operator space, isomorphism between subspaces, Effros-Borel structure, classification

\section{Introduction and main results}

Recently, there has been much progress in describing the complexity of various relations between subspaces of a given separable Banach space. The reader is referred to $[4,5,6]$ for the known results on the relations of isomorphism, biembeddability, and more. Isometry and local equivalence (finite representability) are handled in [16] and [7], respectively.

In this paper, we consider an operator space analogue of this problem (see Section 2 for a brief introduction into operator spaces). The Effros-Borel structure on the set $\mathbf{S}(Z)$ of infinite-dimensional subspaces of a separable operator space $Z$ is defined in the same way as for Banach spaces, see e.g. [11, Chapter 12], or [6]. Reasoning as in Section 2 of [6], we show that the relations of complete isomorphism, complete biembeddability, and such, defined on $\mathbf{S}(Z)$, are analytic equivalence relations.

In the commutative space setting, the famous Gowers-Komorowski-Tomczak Theorem (see e.g. [25]) shows that any separable Banach space, isomorphic to all of its infinite dimensional subspaces, must be isomorphic to $\ell_{2}$. The space $\ell_{2}$ can be equipped with uncountably many 1 -homogeneous operator space structures (an operator space $X$ is called 1-homogeneous if the equality $\|u\|=\|u\|_{c b}$ holds for any $u \in B(X)$ ). For 
instance, the row and column spaces $\mathbf{R}$ and $\mathbf{C}$, as well as their complex interpolation spaces $(\mathbf{R}, \mathbf{C})_{\theta}$, have this property. These, and other, examples of homogeneous Hilbertian spaces can be found, for instance, in [21, 22] (an operator space is called Hilbertian if it is isomorphic to a Hilbert space). Clearly, any 1-homogeneous operator space, having $\ell_{2}$ as its underlying Banach space, is completely isometric to all of its infinite dimensional subspaces. For such spaces, the relation of complete isomorphism between infinite dimensional subspaces is trivial.

It is not known how "simple" the relation of complete isomorphism between subspaces of $X$ may get if $X$ is not homogeneous (or not isomorphic to a Hilbert space). Our main result provides a partial answer to this question.

Theorem 1.1 There exists a family $\mathfrak{F}$ of operator spaces, such that, for any operator space $X \in \mathfrak{F}$, the following is true:

(1) $X$ is isometric to $\ell_{2}$.

(2) The relation of complete isomorphism and complete biembeddability on $\mathbf{S}(X)$ are Borel bireducible to the complete $\mathbf{K}_{\sigma}$ relation.

The family $\mathfrak{F}$ contains a continuum of operator spaces, not completely isomorphic to each other.

It is possible to prove that the relation of complete isometry on $\mathbf{S}(X)(X \in \mathfrak{F})$ is Borel bireducible to the equality on $\mathbb{R}$. The proof proceeds along the same lines as Theorem 6.1, but is exceedingly technical, and not very illuminating. We therefore omit it, and present a related Theorem 1.4 instead.

Each space from $\mathfrak{F}$ has its canonical basis (defined in Section 4), which is 1-completely unconditional. It turns out these bases have interesting properties of their own.

Theorem 1.2 Any subspace $Y$ of an operator space $X$ from the family $\mathfrak{F}$ has 1 completely unconditional canonical basis. Any $C$-completely unconditional basis in such a $Y$ is $\phi(C)$-equivalent (up to a permutation) to the canonical basis of $Y$, with $\phi(C)$ polynomial in $C$.

For certain spaces $X$, a stronger result holds.

Theorem 1.3 For any $a>1$ there exists an operator space $X$, belonging to the family $\mathfrak{F}$, such that the canonical basis in any subspace of $X$ is $a$-equivalent to a subsequence of the canonical basis of $X$. Consequently, every $C$-completely unconditional basic sequence in $X$ is $\phi(C)$-equivalent (up to a permutation) to a subsequence of the canonical basis, with $\phi(C)$ polynomial in $C$. 
Note that homogeneous Hilbertian operator spaces satisfy the conditions of the three theorems above. The spaces from the family $\mathfrak{F}$ are not homogeneous, yet, by Theorems 1.2 and 1.3, they share certain essential properties of homogeneous spaces.

As part of our motivation lies in the field of Banach spaces, we mention a few classical results related to Theorems 1.1, 1.2, and 1.3.

No "commutative" counterpart of Theorem 1.1 has yet been obtained: for a Banach space $E$, very little is known about the "upper" estimates on the complexity of the isomorphism relation on $\mathbf{S}(E)$. It is possible that the relation of isomorphism on $\mathbf{S}(E)$ is complete analytic whenever $E$ is a separable Banach space, not isomorphic to $\ell_{2}$.

Theorem 1.2 shows (among other things) that every subspace of $X$ has an unconditional basis. In the commutative setting, $\ell_{2}$ is the known only separable Banach space with the property that each of its separable subspaces has an unconditional basis. It is a long-standing open question (see e.g. [8, p. 203]) whether there exist other spaces sharing the same property. If a separable $E$ has this property, it must be "close to" $\ell_{2}$. First, any subspace of $E$ has the Approximation Property, therefore, by [15, Theorem 1.g.6], $E$ has to have type $2-\varepsilon$ and cotype $2+\varepsilon$ for any $\varepsilon>0$. Furthermore, by [20, Theorem 10.13], $E$ has weak cotype 2. By [12], $E$ is $\ell_{2}$-saturated. Finally, if $E=\ell_{2}(F)$ for some Banach space $F$, then $E$ is isomorphic to a Hilbert space [13].

Searching for a "commutative" analogue of Theorem 1.3, we strengthen the question posed in the previous paragraph: suppose a Banach space $E$ has an unconditional basis $\left(e_{i}\right)$, such that every subspace of $E$ has an unconditional basis, equivalent to a subsequence of $\left(e_{i}\right)$. Must $E$ be isomorphic to $\ell_{2}$ ?

Finally, in the Banach space setting, we construct a space $E$, not isometric to $\ell_{2}$, but whose subspace structure is "very simple."

Theorem 1.4 The following equivalence relations between infinite dimensional subspaces of $\mathbb{R} \oplus_{1} \ell_{2}$ (or, in the complex case, $\mathbb{C} \oplus_{1} \ell_{2}$ ) are Borel bireducible to $(\mathbb{R},=$ ): (i) isometry, (ii) having Banach-Mazur distance 1; (iii) isometric bi-embeddability; (iv) almost isometric bi-embeddability.

Recall that the Banach-Mazur distance between $Y$ and $Z$ is defined as $d(Y, Z)=$ $\inf \left\{\|u\|\left\|u^{-1}\right\|: u \in B(Y, Z)\right\} . \quad Y$ is called almost isometrically embeddable into $Z$ if for every $\varepsilon>0$ there exists $Y_{\varepsilon} \hookrightarrow Z$ s.t. $d\left(Y, Y_{\varepsilon}\right)<1+\varepsilon(d(\cdot, \cdot)$ denotes the Banach-Mazur distance between spaces).

The rest of the paper is organized as follows: Section 2 provides a brief introduction into operator spaces and c.b. maps. In Section 3 we construct, for each contraction 
$A \in B\left(\ell_{2}\right)$, a subspace $X(A)$ of $\mathbf{R} \oplus \mathbf{C}$, and study the properties of the spaces $X(A)$. Further investigation is carried out in Section 4 , where we concentrate on the case when $A$ is compact. Unconditional bases in the spaces $X(A)$ are described in Section 5. In Section 6 we show that, for the "right" compact contractions $A$, the relations of complete isomorphism and complete biembeddability on $\mathbf{S}(X(A))$ are Borel bireducible to the complete $\mathbf{K}_{\sigma}$ relation. Having gathered all the preliminary results, we prove Theorems 1.1, 1.2, and 1.3 in Section 7. Finally, in Section 8 we prove Theorem 1.4.

\subsection{Acknowledgements}

The first author was partially supported by CORCL grant from the UC system. The second author was partially supported by NSF grants DMS 0901405 and DMS 0919700. We would like to thank the referee for valuable comments, and, in particular, for bringing the reference [8] to our attention.

\section{Introduction into operator spaces}

As our paper deals primarily with operator spaces, we are compelled to recall some basic definitions and facts about the topic. An interested reader is referred to [3, 19, 22] for more information. A (concrete) operator space $X$ is, for us, just a closed subspace of $B(H)$ ( $H$ is a Hilbert space). If $X$ and $Y$ are operator spaces, embedded into $B(H)$ and $B(K)$ respectively, we defined the minimal tensor product of $X$ and $Y$ (denoted simply by $X \otimes Y)$ as the closure of the algebraic tensor product $X \odot Y$ in $B\left(H \otimes_{2} K\right)$. It is common to denote $M_{n} \otimes X$ by $M_{n}(X)$. Here, $M_{n}=B\left(\ell_{2}^{n}\right)$ is the space of $n \times n$ matrices. We view $M_{n}(X)$ as the space of $X$-valued $n \times n$ matrices, with the norm $\|\cdot\|_{n}$. It is easy to see that the sequence of matricial norms $\|\cdot\|_{n}$ satisfies two properties (Ruan's axioms): (i) for any $v \in M_{n}(X), \alpha \in M_{n, k}$, and $\beta \in M_{k, n}$, $\left\|\left(\beta \otimes I_{X}\right) v\left(\alpha \otimes I_{X}\right)\right\|_{k} \leqslant\|\beta\|\|x\|_{n}\|\alpha\|$, and (ii) for any $v \in M_{n}(X)$ and $w \in M_{k}(X) \|$, $\|v \oplus w\|_{k+n}=\max \left\{\|v\|_{n},\|w\|_{k}\right\}$. It turns out that the converse is also true. Suppose $X$ is a matricially normed space - that is, it is a Banach space, for which the spaces $M_{n}(X)$ of $n \times n X$-valued matrices are equipped with the norms $\|\cdot\|_{n}$, satisfying (i) and (ii) above. Then the norms $\|\cdot\|_{n}$ arise from an isometric embedding of $X$ into $B(H)$, for some Hilbert space $H$. The spaces $X$ as above are sometimes called abstract operator spaces.

It is easy to see that a subspace of an operator space is, again, an operator space (we use the notation $\hookrightarrow$ to denote one operator space being a subspace of another). Moreover, 
a quotient and the dual of an operator space can be again equipped with an operator space structure. Once again, the reader is referred to $[3,19,22]$ for details.

A map $u$ from an operator space $X$ to an operator space $Y$ is called completely bounded (c.b. for short) if its c.b. norm

$$
\|u\|_{c b}=\sup _{n}\left\|I_{M_{n}} \otimes u\right\|_{B\left(M_{n}(X), M_{n}(Y)\right)}=\left\|I_{B\left(\ell_{2}\right)} \otimes u\right\|_{B\left(B\left(\ell_{2}\right) \otimes X, B\left(\ell_{2}\right) \otimes Y\right)}
$$

is finite. The set of all c.b. maps from $X$ to $Y$ is denoted by $C B(X, Y)$. Clearly, $\|u\|_{c b} \geq\|u\|$, and $C B(X, Y) \subset B(X, Y)$ (the inclusion may be strict). The operator spaces $X$ and $Y$ are called completely isomorphic (completely isometric) if there exists $u \in C B(X, Y)$ such that $u^{-1}$ is c.b. (resp. $\|u\|_{c b}=\left\|u^{-1}\right\|_{c b}=1$ ). We shall use the notation $\simeq$ for complete isomorphism. Moreover, we say that $X$ is c-completely isomorphic to $Y(X \stackrel{c}{\simeq} Y)$ if there exists $u \in C B(X, Y)$ with $\|u\|_{c b}\left\|u^{-1}\right\|_{c b} \leqslant c$. Complete embeddability and biembeddability are defined in the obvious way.

Suppose the operator space s $X$ and $Y$ are embedded into $B(H)$ and $B(K)$, respectively. We define the direct sum of operator spaces $X$ and $Y$ (denoted by $X \oplus_{\infty} Y$, or simply $X \oplus Y)$ by viewing $X \oplus Y$ as embedded into $B\left(H \oplus_{2} K\right)$. Note that any $u \in M_{n}(X \oplus Y)$ has a unique expansion as $v \oplus w$, with $v \in M_{n}(X)$ and $w \in M_{n}(Y)$. Then $\|u\|=\max \{\|v\|,\|w\|\}$.

Throughout this paper, we work with the row and column spaces. Recall that a Hilbert space $H$ can be equipped with row and column operator space structure, denoted by $H_{\mathbf{R}}$ and $H_{\mathbf{C}}$, respectively. The space $H_{\mathbf{R}}$ is defined as the linear space of operators $\xi \otimes \xi_{0}$, where $\xi_{0}$ is a fixed unit vector, and $\xi$ runs over $H$. Here, for $\xi \in H$ and $\eta \in K$, $\xi \otimes \eta$ denotes the operator in $B(H, K)$, defined by $(\xi \otimes \eta) \zeta=\langle\zeta, \xi\rangle \eta$. Similarly, the space $H_{\mathbf{C}}$ is defined as the space of operators $\xi_{0} \otimes \xi(\xi \in H)$.

It is easy to see that, if $K$ is a subspace of $H$, then $K_{\mathbf{C}}\left(K_{\mathbf{R}}\right)$ is a subspace of $H_{\mathbf{C}}$ (resp. $\left.H_{\mathbf{R}}\right)$. For simplicity of notation, we write denoted by $\mathbf{R}$ and $\mathbf{C}$, instead of $\left(\ell_{2}\right)_{\mathbf{R}}$ and $\left(\ell_{2}\right)_{\mathbf{C}}$, respectively. One can use matrix units to describe these spaces. We denote by $E_{i j} \in B\left(\ell_{2}\right)$ the infinite matrix with 1 on the intersection of the $i$-th row and the $j$-th column, and zeroes elsewhere. Then $\mathbf{R}(\mathbf{C})$ is the closed linear span of $\left(E_{1 j}\right)_{j=1}^{\infty}$ (respectively, $\left.\left(E_{i 1}\right)_{i=1}^{\infty}\right)$ ). Below we list a few useful properties of row and column spaces. Here, $H$ and $K$ are Hilbert spaces.

(1) $H_{\mathbf{R}}$ and $H_{\mathbf{C}}$ are isometric to $H$ (as Banach spaces).

(2) For any $u \in B(H, K),\|u\|=\|u\|_{C B\left(H_{\mathbf{R}}, K_{\mathbf{R}}\right)}=\|u\|_{C B\left(H_{\mathbf{C}}, K_{\mathbf{C}}\right)}$. 
(3) If $\left(\xi_{i}\right)$ is an orthonormal system in $H$, then, for any finite sequence $\left(a_{i}\right)$ of elements of $M_{n}$,

$$
\begin{aligned}
& \left\|\sum_{i} a_{i} \otimes \xi_{i}\right\|_{M_{n}\left(H_{\mathbf{R}}\right)}=\left\|\left(\sum_{i} a_{i} a_{i}^{*}\right)^{1 / 2}\right\|=\left\|\sum_{i} a_{i} a_{i}^{*}\right\|^{1 / 2}, \\
& \left\|\sum_{i} a_{j} \otimes \xi_{i}\right\|_{M_{n}\left(H_{\mathbf{C}}\right)}=\left\|\left(\sum_{i} a_{i}^{*} a_{i}\right)^{1 / 2}\right\|=\left\|\sum_{i} a_{i}^{*} a_{i}\right\|^{1 / 2} .
\end{aligned}
$$

(4) Suppose $H^{(1)}, \ldots, H^{(n)}$ are Hilbert spaces. Then the formal identity map id : $\left(H^{(1)} \oplus_{2} \ldots \oplus_{2} H^{(n)}\right)_{\mathbf{R}} \rightarrow H_{\mathbf{R}}^{(1)} \oplus \ldots \oplus H_{\mathbf{R}}^{(n)}$ is a complete contraction, and $\left\|i d^{-1}\right\|_{c b} \leqslant \sqrt{n}$. The same is also true for column spaces.

(5) For any $u \in C B\left(H_{\mathbf{R}}, K_{\mathbf{C}}\right)$ or $u \in C B\left(H_{\mathbf{C}}, K_{\mathbf{R}}\right),\|u\|_{2}=\|u\|_{c b}$ (here, $\|\cdot\|_{2}$ is the Hilbert-Schmidt norm).

(6) Duality: $H_{\mathbf{R}}^{*}=H_{\mathbf{C}}$, and $H_{\mathbf{C}}^{*}=H_{\mathbf{R}}$.

(7) For any operator space $X$ and any $u \in B\left(X, H_{\mathbf{C}}\right)\left(u \in B\left(X, H_{\mathbf{R}}\right)\right),\|u\|_{c b}=$ $\left\|I_{\mathbf{C}} \otimes u\right\|_{B\left(\mathbf{C} \otimes X, \mathbf{C} \otimes H_{\mathbf{C}}\right)}$ (resp. $\left.\|u\|_{c b}=\left\|I_{\mathbf{R}} \otimes u\right\|_{B\left(\mathbf{R} \otimes X, \mathbf{R} \otimes H_{\mathbf{R}}\right)}\right)$. This result follows from the proof of Smith's lemma - see e.g. Proposition 2.2.2 of [3].

(8) If $H$ is separable infinite dimensional, then $H_{\mathbf{R}}\left(H_{\mathbf{C}}\right)$ is completely isometric to R (resp. C).

A sequence $\left(x_{i}\right)_{i \in I}$ in an operator space $X$ is called normalized if $\left\|x_{i}\right\|=1$ for every $i \in I .\left(x_{i}\right)$ is said to be a $c$-completely unconditional basic sequence $(c \geq 1)$ if, for any finite sequence of matrices $\left(a_{i}\right)$, and any sequence of scalars $\lambda_{i} \in\{\lambda \in \mathbb{C}:|\lambda| \leqslant 1\}$, we have $\left\|\sum_{i} \lambda_{i} a_{i} \otimes x_{i}\right\|_{M_{n}(X)} \leqslant c\left\|\sum_{i} a_{i} \otimes x_{i}\right\|_{M_{n}(X)}$. It is easy to see that any such sequence $\left(x_{i}\right)$ is linearly independent. A $c$-completely unconditional basic sequence $\left(x_{i}\right) \subset X$ is called a c-completely unconditional basis in $X$ if $X=\operatorname{span}\left[x_{i}: i \in I\right]$ (here and below, $\operatorname{span}[\mathcal{F}]$ refers to the closed linear span of the family $\mathcal{F}$ ).

A convenient example of a normalized 1-completely unconditional basis is provided by an orthonormal basis in $\mathbf{R}$ or $\mathbf{C}$. For future reference, we observe that any completely unconditional basis is "similar to" an orthogonal one. More precisely, suppose $X$ is an operator space, which is isometric to a Hilbert space (this is the setting we are concerned with in this paper). Suppose, furthermore, that $\left(x_{i}\right)$ is a normalized $c$ completely unconditional basis in an operator space $X$, isometric to a Hilbert space, then $\left(x_{i}\right)$ is "similar to" an orthonormal basis: for any finite sequence of scalars $\left(\alpha_{i}\right)$,

$$
c^{2}\left\|\sum_{i} \alpha_{i} x_{i}\right\|^{2} \geq \operatorname{Ave}_{ \pm}\left\|\sum_{i} \pm \alpha_{i} x_{i}\right\|^{2}=\sum_{i}\left|\alpha_{i}\right|^{2},
$$

and similarly, $c^{-2}\left\|\sum_{i} \alpha_{i} x_{i}\right\|^{2} \leqslant \sum_{i}\left|\alpha_{i}\right|^{2}$. Thus, there exists an $U: X \rightarrow \ell_{2}(I)$, s.t. $\left(U x_{i}\right)_{i \in I}$ is an orthonormal basis, and $\|U\|,\left\|U^{-1}\right\| \leqslant c$.

Families $\left(x_{i}\right)_{i \in I}$ and $\left(y_{i}\right)_{i \in I}$ in operator spaces $X$ and $Y$, respectively, are called $c$ equivalent if there exists a map $u: \operatorname{span}\left[x_{i}: i \in I\right] \rightarrow \operatorname{span}\left[y_{i}: i \in I\right]$, such 
that $u x_{i}=y_{i}$, and $\|u\|_{c b}\left\|u^{-1}\right\|_{c b} \leqslant c$. Two sequences are equivalent if they are $c$ -

equivalent, for some $c$. Furthermore, $\left(x_{i}\right)_{i \in I}$ is $c$-equivalent to a subfamily of $\left(y_{j}\right)_{j \in J}$ if there exists a subset $I^{\prime} \subset J$, such that $|I|=\left|I^{\prime}\right|$, and $\left(x_{i}\right)_{i \in I}$ is $c$-equivalent to $\left(y_{j}\right)_{j \in I^{\prime}}$. Finally, $\left(x_{i}\right)_{i \in I}$ is c-equivalent to $\left(y_{i}\right)_{i \in I}$ up to a permutation if there exists a bijection $\pi: I \rightarrow I$ such that $\left(x_{i}\right)_{i \in I}$ is $c$-equivalent to $\left(y_{\pi(i)}\right)_{i \in I}$.

A sequence $\left(x_{i}\right)_{i \in I}$ is called completely unconditional if it is $c$-completely unconditional, for some $c$. A completely unconditional basis, equivalence of sequences etc. are defined by dropping the $c$, in a similar manner.

\section{Subspaces of $\mathbf{R} \oplus \mathbf{C}$ : basic facts}

Suppose $H$ and $K$ are separable Hilbert spaces, and $A \in B(H, K)$ is a contraction. Denote by $X_{\mathbf{R}}(H, K, A)$ the subspace of $H_{\mathbf{R}} \oplus K_{\mathbf{C}}$, spanned by $(e, A e)(e \in H)$. If there is no confusion as to the spaces $H$ and $K$, we simply write $X_{\mathbf{R}}(A) . X_{\mathbf{C}}(A)$ is defined in the same way. Often, we write $X(A)(X(H, K, A))$ instead of $X_{\mathbf{R}}(A)\left(X_{\mathbf{R}}(H, K, A)\right)$. Note that the formal identity map $i d: H \rightarrow X(H, K, A): \xi \mapsto \xi \oplus A \xi$ is an isometry. Thus, we identify subspaces of $X(H, K, A)$ with those of $H$. This identification gives meaning to the notation $\left.A\right|_{Y}$, where $Y \hookrightarrow X(A)$.

Remark 3.1 Although the spaces $\mathbf{R}$ and $\mathbf{C}$ are "simple", the structure of their direct sum $\mathbf{R} \oplus C$ is rather rich. For instance, it was shown in [27] that the "operator Hilbert space" $O H$ is a subspace of a quotient of $\mathbf{R} \oplus \mathbf{C}$ (actually, the results of that paper are much more general). It follows from [27] that the spaces $X(A)\left(A \in B\left(\ell_{2}\right)\right)$ defined as above are natural "building blocks" of subspaces of $\mathbf{R} \oplus \mathbf{C}$.

Begin by stating a simple lemma.

Lemma 3.2 Suppose $H, K$, and $K^{\prime}$ are Hilbert spaces, and $A \in B(H, K)$ is a contraction.

(1) Suppose $U \in B\left(K, K^{\prime}\right)$ is such that $\|U \xi\|=\|\xi\|$ for any $\xi \in \operatorname{ran} A$. Then $X(H, K, A)$ is completely isometric to $X\left(H, K^{\prime}, U A\right)$. In particular, $X(H, K, A)$ is completely isometric to $X(H, H,|A|)$, where $|A|=\left(A^{*} A\right)^{1 / 2}$.

(2) Suppose $P_{1}, \ldots, P_{m}$ and $Q_{1}, \ldots, Q_{m}$ are families of orthogonal mutually orthogonal projections on $H$ and $K$, respectively, such that $\sum_{k} P_{k}=I_{H}, \sum_{k} Q_{k}=I_{K}$, and $A=\sum_{k=1}^{m} Q_{k} A P_{k}$. Set $H_{k}=P_{k}(H)$ and $K_{k}=Q_{k}(K)$. Then the formal identity operator id from $X(H, K, A)$ to $X\left(H_{1}, K_{1}, Q_{1} A P_{1}\right) \oplus \ldots \oplus$ $X\left(H_{m}, K_{m}, Q_{m} A P_{m}\right)$ is a complete contraction, and $\left\|i d^{-1}\right\|_{c b} \leqslant \sqrt{m}$. 
(3) $X(A)$ is $|\lambda|^{-1}$-completely isomorphic to $X(\lambda A)$ whenever $0<|\lambda| \leqslant 1$.

Proof We only establish part (2). A Gram-Schmidt orthogonalization shows that any element $x \in M_{n}(H)$ can be written as $x=x_{1}+\ldots+x_{n}$, with $x_{k}=\sum_{i} a_{k i} \otimes \xi_{k i}$ $a_{k i} \in M_{n}$, and $\left(\xi_{k i}\right)$ a finite orthonormal systems in $H_{k}$. Then

$$
\|x\|_{M_{n}(X(A))}=\max \left\{\left\|\sum_{k} \sum_{i} a_{k i} \otimes \xi_{k i}\right\|_{M_{n}\left(H_{\mathbf{R}}\right)},\left\|\sum_{k} \sum_{i} a_{k i} \otimes A \xi_{k i}\right\|_{M_{n}\left(K_{\mathbf{C}}\right)}\right\} .
$$

By the basic properties of row and column spaces (listed at the end of the previous section),

$$
\begin{aligned}
\max _{k}\left\|\sum_{i} a_{k i} \otimes \xi_{k i}\right\|_{M_{n}\left(H_{\mathbf{R}}\right)} & \leqslant\left\|\sum_{k, i} a_{k i} \otimes \xi_{k i}\right\|_{M_{n}\left(H_{\mathbf{R}}\right)} \\
& \leqslant \sqrt{m} \max _{k}\left\|\sum_{i} a_{k i} \otimes \xi_{k i}\right\|_{M_{n}\left(H_{\mathbf{R}}\right)} .
\end{aligned}
$$

Furthermore, the vectors $A \xi_{k i}$ belong to the mutually orthogonal spaces $K_{k}$, hence

$$
\begin{aligned}
\max _{k}\left\|\sum_{i} a_{k i} \otimes A \xi_{k i}\right\|_{M_{n}\left(H_{\mathbf{C}}\right)} & \leqslant\left\|\sum_{k, i} a_{k i} \otimes A \xi_{k i}\right\|_{M_{n}\left(H_{\mathbf{C}}\right)} \\
& \leqslant \sqrt{m} \max _{k}\left\|\sum_{i} a_{k i} \otimes A \xi_{k i}\right\|_{M_{n}\left(H_{\mathbf{C}}\right) .}
\end{aligned}
$$

Note that

$$
\begin{aligned}
& \left\|x_{k}\right\|_{M_{n}\left(X\left(H_{k}, Q_{k}, Q_{k} A P_{k}\right)\right)} \\
& =\max \left\{\left\|\sum_{i} a_{k i} \otimes \xi_{k i}\right\|_{M_{n}\left(H_{\mathbf{R}}\right)},\left\|\sum_{i} a_{k i} \otimes A \xi_{k i}\right\|_{M_{n}\left(H_{\mathbf{C}}\right)}\right\} .
\end{aligned}
$$

Therefore,

$$
\begin{aligned}
& \max _{k}\left\|x_{k}\right\|_{M_{n}\left(X\left(H_{k}, Q_{k}, Q_{k} A P_{k}\right)\right)} \\
& =\max \left\{\max _{k}\left\|\sum_{i} a_{k i} \otimes \xi_{k i}\right\|_{M_{n}\left(H_{\mathbf{R}}\right)}, \max _{k}\left\|\sum_{i} a_{k i} \otimes A \xi_{k i}\right\|_{M_{n}\left(H_{\mathbf{C}}\right)}\right\} \\
& \leqslant\|x\|_{M_{n}(X(A))} \\
& \leqslant \sqrt{m} \max \left\{\max _{k}\left\|\sum_{i} a_{k i} \otimes \xi_{k i}\right\|_{M_{n}\left(H_{\mathbf{R}}\right)}, \max _{k}\left\|\sum_{i} a_{k i} \otimes A \xi_{k i}\right\|_{M_{n}\left(H_{\mathbf{C}}\right)}\right\} .
\end{aligned}
$$

We complete the proof by noting that

$$
\|x\|_{M_{n}\left(X\left(H_{1}, K_{1}, Q_{1} A P_{1}\right) \oplus \ldots \oplus X\left(H_{m}, K_{m}, Q_{m} A P_{m}\right)\right)}=\max _{1 \leqslant k \leqslant m}\left\|x_{k}\right\|_{M_{n}\left(X\left(H_{k}, Q_{k}, Q_{k} A P_{k}\right)\right)} .
$$

Next, we establish our key tool for computing c.b. norms. 
Lemma 3.3 Suppose $A$ and $B$ are contractions, and $T \in B(X(A), X(B))$. Then

$$
\|T\|_{c b}=\max \left\{\|T\|, \sup \left\{\|B T u\|_{2}: u \in B\left(\ell_{2}, X(A)\right),\|A u\|_{2} \leqslant 1,\|u\| \leqslant 1\right\}\right\} .
$$

Proof By definition, $\|T\|_{c b}=\max \left\{\|T\|_{C B(X(A), \mathbf{R})},\|B T\|_{C B(X(A), \mathbf{C})}\right\}$. To estimate the first term, note that id $: X(A) \rightarrow \mathbf{R}$ is a complete contraction, hence

$$
\|T\|_{C B(X(A), \mathbf{R})}=\|T \circ i d\|_{C B(X(A), \mathbf{R})} \leqslant\|T\|_{C B(\mathbf{R}, \mathbf{R})}\|i d\|_{C B(X(A), \mathbf{R})}=\|T\| .
$$

However, $\|T\| \leqslant\|T\|_{C B(X(A), \mathbf{R})}$, hence $\|T\|=\|T\|_{C B(X(A), \mathbf{R})}$.

Next we estimate $\|B T\|_{C B(X(A), \mathbf{C})}$. We know that

$$
\|B T\|_{C B(X(A), \mathbf{C})}=\sup \left\{\left\|\left(I_{\mathbf{C}} \otimes B T\right)(x)\right\|_{\mathbf{C} \otimes \mathbf{C}}: x \in \mathbf{C} \otimes X(A),\|x\|_{\mathbf{C} \otimes X(A)} \leqslant 1\right\} .
$$

Identifying elements of $\mathbf{C} \otimes X(A)$ and $\mathbf{C} \otimes \mathbf{C}$ with operators from $\mathbf{R}$ to $X(A)$ and $\mathbf{C}$, respectively, we see that

$$
\|B T\|_{C B(X(A), \mathbf{C})}=\sup \left\{\|B T u\|_{2}: u \in C B(\mathbf{R}, X(A)),\|u\|_{c b} \leqslant 1\right\} .
$$

But $\|u\|_{c b}=\max \left\{\|u\|_{C B(\mathbf{R})},\|A u\|_{C B(\mathbf{R}, \mathbf{C})}\right\}=\max \left\{\|u\|,\|A u\|_{2}\right\}$.

Next we examine the exactness of $X(A)^{*}$. Recall that an operator space $X$ is called exact if there exists $c>0$ such that, for any finite dimensional subspace $E$ of $X$, there exists an operator $u$ from $E$ to a subspace $F$ of $M_{n}(n \in \mathbb{N})$, such that $\|u\|_{c b}\left\|u^{-1}\right\|_{c b}<c$. The infimum of all the $c$ 's like this is called the exactness constant of $X$. It is easy to see that $\mathbf{R} \oplus \mathbf{C}$ is 1 -exact, hence so is $X(A)$. The case of the dual is different.

Proposition 3.4 Suppose $A \in B(H, K)$ is a contraction. Then the exactness constant of $X(A)^{*}$ is at least $2^{-5 / 2}\|A\|_{2}$. In particular, $X(A)^{*}$ is not exact if $A$ is not HilbertSchmidt.

Proof Let $c=2^{5 / 2} \operatorname{ex}\left(X(A)^{*}\right)$. By Corollary 0.7 of [23], there exist operators $T_{1}$ : $X(A) \rightarrow \mathbf{R}, T_{2}: \mathbf{R} \rightarrow X(A), S_{1}: X(A) \rightarrow \mathbf{C}$, and $T_{2}: \mathbf{C} \rightarrow X(A)$, such that $i d=S_{2} S_{1}+T_{2} T_{1}$ (id is the identity on $X(A)$ ), and

$$
\left\|S_{1}\right\|_{c b}\left\|S_{2}\right\|_{c b}+\left\|T_{1}\right\|_{c b}\left\|T_{2}\right\|_{c b} \leqslant \max \left\{\left\|S_{1}\right\|_{c b},\left\|T_{1}\right\|_{c b}\right\}\left(\left\|S_{2}\right\|_{c b}+\left\|T_{2}\right\|_{c b}\right) \leqslant c
$$

By Lemma 3.3, $\left\|T_{2}\right\|_{c b} \geq\left\|A T_{2}\right\|_{2}$, and a simple calculation yields

$$
\left\|S_{2}\right\|_{c b} \geq\left\|S_{2}\right\|_{C B(\mathbf{C}, \mathbf{R})}=\left\|S_{2}\right\|_{2} \geq\left\|A S_{2}\right\|_{2} .
$$

Therefore,

$$
\begin{aligned}
\left\|S_{1}\right\|_{c b}\left\|S_{2}\right\|_{c b}+\left\|T_{1}\right\|_{c b}\left\|T_{2}\right\|_{c b} & \geq\left\|S_{1}\right\|\left\|A S_{2}\right\|_{2}+\left\|T_{1}\right\|\left\|A T_{2}\right\|_{2} \\
& \geq\left\|A\left(S_{2} S_{1}+T_{2} T_{1}\right)\right\|_{2}=\|A\|_{2},
\end{aligned}
$$

which implies the desired estimate for $c$. 
Corollary 3.5 The space $X(A)$ is completely isomorphic to $\mathbf{R}$ if and only if $A$ is Hilbert-Schmidt. Furthermore, the formal identity map id $: \mathbf{R} \rightarrow X(A)$ is completely contractive, and $\left\|i d^{-1}\right\|_{c b}=\max \left\{1,\|A\|_{2}\right\}$.

Proof The estimates on the c.b. norm of $i d$ and $i d^{-1}$ follow from Lemma 3.3. Thus, $X(A) \stackrel{\max \left\{1,\|A\|_{2}\right\}}{\simeq} \mathbf{R}$ whenever $A$ is Hilbert-Schmidt. On the other hand, if $A$ is not Hilbert-Schmidt, then, by Proposition $3.4, X(A)^{*}$ is not exact. However, $\mathbf{R}^{*}=\mathbf{C}$ is exact, thus $X(A)$ is not completely isomorphic to $\mathbf{R}$.

We say that $A \in B(H, K)$ ( $H$ and $K$ are Hilbert spaces) is diagonalizable if the eigenvectors of $|A|=\left(A^{*} A\right)^{1 / 2}$ span $H$. Equivalently, there exist orthonormal systems $\left(\xi_{i}\right)$ and $\left(\eta_{i}\right)$ in $H$ and $K$, respectively, and a sequence $\left(\lambda_{i}\right)$ of non-negative numbers, such that $A=\sum_{i} \lambda_{i} \xi_{i} \otimes \eta_{i}$.

When working with $X(A)$, it is often convenient to have $A$ diagonalizable. While every compact operator is diagonalizable, a non-compact one need not have this property. However, we have:

Lemma 3.6 Suppose $H$ and $K$ are separable Hilbert spaces, $A \in B(H, K)$ is a contraction, and $\varepsilon>0$. Then there exists a diagonalizable contraction $B \in B(H, K)$ such that $\|A-B\|_{2} \leqslant \varepsilon$, and $X(A)$ is $(1+\varepsilon)^{2}$-completely isomorphic to $X(B)$. If $A$ is non-negative, $B$ can be selected to be non-negative, too.

Proof By Lemma 3.2(1), it suffices to consider the case of $A=|A|$, and $H=K$. By [26], there exists a selfadjoint diagonalizable $C \in B(H)$ such that $\|A-C\|_{2}<\varepsilon / 2$. Let $\left(c_{i}\right)$ be the eigenvalues of $C$, and $\left(\xi_{i}\right)$ the corresponding norm 1 eigenvectors. Define the operator $B$ by setting $B \xi_{i}=b_{i} \xi_{i}$, where

$$
b_{i}= \begin{cases}c_{i} & 0 \leqslant c_{i} \leqslant 1 \\ 0 & c_{i}<0 \\ 1 & c_{i}>1\end{cases}
$$

We claim that $\|B-C\|_{2}<\varepsilon / 2$. Indeed, let $\mathcal{I}=\left\{i: b_{i} \neq c_{i}\right\}$. Then

$$
\|B-C\|_{2}^{2}=\sum_{i \in \mathcal{I}}\left|b_{i}-c_{i}\right|^{2}=\sum_{i: c_{i}<0}\left|c_{i}\right|^{2}+\sum_{i: c_{i}>1}\left|b_{i}\right|^{2} .
$$

But $\left\langle\xi_{i}, A \xi_{i}\right\rangle \in[0,1]$, hence, for $c_{i}<0,\left|\left\langle\xi_{i}, A \xi_{i}\right\rangle-\left\langle\xi_{i}, C \xi_{i}\right\rangle\right| \geq\left|c_{i}\right|$. Similarly, for $c_{i}>1,\left|\left\langle\xi_{i}, A \xi_{i}\right\rangle-\left\langle\xi_{i}, C \xi_{i}\right\rangle\right| \geq\left|1-c_{i}\right|$. Thus,

$$
\frac{\varepsilon^{2}}{4}>\|A-C\|_{2}^{2}=\sum_{i, j}\left|\left\langle\xi_{i},(A-C) \xi_{j}\right\rangle\right|^{2} \geq \sum_{i}\left|\left\langle\xi_{i},(A-C) \xi_{i}\right\rangle\right|^{2} \geq \sum_{i \in \mathcal{I}}\left|b_{i}-c_{i}\right|^{2} .
$$


By the triangle inequality, $\|A-B\|_{2}<\varepsilon$. Moreover, $B$ is a non-negative contraction.

Denote the the formal identity map from $X(A)$ to $X(B)$ by $U$. It remains to show that $\|U\|_{c b},\left\|U^{-1}\right\|_{c b} \leqslant 1+\varepsilon$. As $U$ is an isometry, Lemma 3.3 implies

$$
\|U\|_{c b}=\sup \left\{\|B u\|_{2}: u \in B\left(\ell_{2}, X(A)\right),\|A u\|_{2} \leqslant 1,\|u\| \leqslant 1\right\} .
$$

By the triangle inequality,

$$
\|B u\|_{2} \leqslant\|A u\|_{2}+\|(B-A) u\|_{2} \leqslant\|A u\|_{2}+\|B-A\|_{2} \leqslant 1+\varepsilon,
$$

hence (3-1) yields $\|U\|_{c b} \leqslant 1+\varepsilon .\left\|U^{-1}\right\|_{c b}$ is estimated similarly.

Proposition 3.7 Suppose $H$ and $K$ are separable Hilbert spaces, $B \in B(H, K)$ is a contraction, and $0 \in \sigma_{\text {ess }}(|B|)$. Then $X(B)$ is $4 \sqrt{2}$-completely isomorphic to $X(B) \oplus \mathbf{R}$.

Proof We can assume $B=|B|$. By Lemma 3.6, there exists a non-negative diagonalizable contraction $A$ such that $A-B$ is Hilbert-Schmidt, and $X(A) \stackrel{2^{1 / 4}}{\simeq} X(B)$. As the essential spectrum is stable under compact perturbations, $0 \in \sigma_{\text {ess }}(A)$. Write $A=\operatorname{diag}(\alpha)$, where $\alpha=\left(\alpha_{i}\right)_{i \in I}$, and $\operatorname{diag}(\alpha) \in B\left(\ell_{2}\right)$ is the diagonal operator with $\alpha, \alpha_{2}, \ldots$ on the main diagonal. Then 0 is a cluster point of the set $\left(\alpha_{i}\right)$. Denote the norm 1 eigenvectors, corresponding to $\alpha_{i}$, by $\xi_{i}$. Find $I_{0} \subset I$ such that $\sum_{i \in I_{0}} \alpha_{i}^{2}<1$. Let $I_{1}=I \backslash I_{0}$, and let $P_{0}$ and $P_{1}$ be the orthogonal projections onto $H_{0}=\operatorname{span}\left[\xi_{i}: i \in I_{0}\right]$ and $H_{1}=\operatorname{span}\left[\xi_{i}: i \in I_{1}\right]$, respectively. By Lemma 3.2, $X(A)$ is $\sqrt{2}$-completely isomorphic to $X\left(H_{0}, H_{0}, P_{0} A P_{0}\right) \oplus X\left(H_{1}, H_{1}, P_{1} A P_{1}\right)$. By Corollary $3.5, X\left(H_{0}, H_{0}, P_{0} A P_{0}\right)$ is completely isometric to $\mathbf{R}$. Thus,

$$
\begin{aligned}
& X(A) \stackrel{\sqrt{2}}{\simeq} X\left(H_{0}, H_{0}, P_{0} A P_{0}\right) \oplus X\left(H_{1}, H_{1}, P_{1} A P_{1}\right) \\
& \stackrel{\sqrt{2}}{\simeq} \mathbf{R} \oplus\left(H_{1}, H_{1}, P_{1} A P_{1}\right) \stackrel{\sqrt{2}}{\simeq} \mathbf{R} \oplus \mathbf{R} \oplus X\left(H_{1}, H_{1}, P_{1} A P_{1}\right) \\
& =\mathbf{R} \oplus X\left(H_{0}, H_{0}, P_{0} A P_{0}\right) \oplus X\left(H_{1}, H_{1}, P_{1} A P_{1}\right) \stackrel{\sqrt{2}}{\simeq} \mathbf{R} \oplus X(A) .
\end{aligned}
$$

To summarize, $X(A) \stackrel{4}{\simeq} X(A) \oplus \mathbf{R}$. As $X(A) \stackrel{2^{1 / 4}}{\simeq} X(B)$, we are done.

Corollary 3.8 Suppose $H$ and $K$ are separable infinite dimensional Hilbert spaces, and a contraction $A \in B(H, K)$ satisfies $0 \in \sigma_{\text {ess }}(|A|)$. Consider the operator $\tilde{A}=A \oplus 0$ from $\tilde{H}=H \oplus_{2} \ell_{2}$ to $\tilde{K}=K \oplus_{2} \ell_{2}$. Then $X(A) \stackrel{8}{\simeq} X(\tilde{A})$.

Proof Let $P$ be the orthogonal projection from $\tilde{H}$ onto $H$. onto $H$. By Lemma 3.2(2) and Corollary 3.5, $X(\tilde{A}) \stackrel{\sqrt{2}}{\simeq} X(A) \oplus \mathbf{R}$. However, by Proposition 3.7, $X(A) \stackrel{4 \sqrt{2}}{\simeq}$ $X(A) \oplus \mathbf{R}$. 


\section{Classification of subspaces using sequences}

In this section, we study the spaces $X(A)$ when $A$ is compact, and establish a connection between such spaces and a certain family of sequences. We denote by $\mathfrak{C}$ the space of compact contractions $A \in B\left(\ell_{2}\right)$, which are not Hilbert-Schmidt. We denote by $\mathfrak{F}$ the set of all spaces $X(A)$, with $A \in \mathfrak{C}$.

Start by defining the canonical basis in $X(A)$, where $A \in B(H, K)$ is a compact contraction. As $X(A)=X(|A|)$, we assume henceforth that $A=|A|$, and $H=K$. Let $\left(\xi_{i}\right)_{i \in I_{1}}$ be the normalized eigenvectors of $A$, corresponding to the positive eigenvalues of $A$. Furthermore, set $H^{\prime}=\operatorname{ker} A$, and let $\left(\xi_{i}\right)_{i \in I_{0}}$ be an orthonormal basis in $H^{\prime}$ (we assume that $\left.I_{1} \cap I_{0}=\emptyset\right)$. Let $I=I_{0} \cup I_{1}$. The vectors $e_{i}=\xi_{i} \oplus A \xi_{i} \in H_{\mathbf{R}} \oplus H_{\mathbf{C}}(i \in I)$ span $X(A)$. Moreover, $\left\|e_{i}\right\|=1$ for each $i$, and, for any finite sequence $\left(a_{i}\right) \subset M_{n}$,

$$
\left\|\sum_{i} a_{i} \otimes e_{i}\right\|_{M_{n}(X(A))}^{2}=\max \left\{\left\|\sum_{i} a_{i} a_{i}^{*}\right\|,\left\|\sum_{i}\right\| A \xi_{i}\left\|^{2} a_{i}^{*} a_{i}\right\|\right\} .
$$

We say that the vectors $e_{i}=e_{i}[A]$ form the canonical basis of $X(A)$.

Now suppose $\left(\alpha_{i}\right)_{i \in \mathbb{N}}$ is a sequence of numbers in $[0,1]$. In an effort to link operator spaces with certain sequences of scalars, we define the operator space $X^{d}(\alpha)=$ $X(\operatorname{diag}(\alpha))$. To describe the operator space structure of $X^{d}(\alpha)$, denote by $\left(\xi_{i}\right)$ the canonical orthonormal basis of $\ell_{2}$. Then the vectors $e_{i}(\alpha)=e_{i}=\xi_{i} \oplus \alpha_{i} \xi_{i} \in H_{\mathbf{R}} \oplus K_{\mathbf{C}}$ form a 1 -completely unconditional orthonormal basis in $X^{d}(\alpha)$, with

$$
\left\|\sum_{i} a_{i} \otimes e_{i}\right\|^{2}=\max \left\{\left\|\sum_{i} a_{i} a_{i}^{*}\right\|,\left\|\sum_{i} \alpha_{i}^{2} a_{i}^{*} a_{i}\right\|\right\}
$$

for any finite sequence of matrices $\left(a_{i}\right)$. We call $\left(e_{i}(\alpha)\right)_{i \in \mathbb{N}}$ the canonical basis of $X^{d}(\alpha)$. The formal identity from $X^{d}(\alpha)$ to $X^{d}(\beta)$ is the linear operator mapping $e_{i}(\alpha)$ to $e_{i}(\beta)$.

If $\alpha=\left(\alpha_{i}\right)_{i=1}^{n}$ is a finite sequence, we define the finite dimensional space $X^{d}(\alpha)$ in the same way.

To reduce ourselves to working with the spaces $X^{d}(\alpha)$ (and hence to sequences of scalars), define, for a compact $A \in B(H, K)$, the sequence $\alpha=\mathbf{D}(A)$ : if $A$ has rank $n<\infty$, let $\alpha_{1} \geq \ldots \geq \alpha_{n}>0$ be the non-zero singular values of $A$, and set $\alpha_{i}=0$ for $i>n$. If the rank of $A$ is infinite, let $\left(\alpha_{i}\right)$ be the singular values of $A$, listed in the non-increasing order. We have:

Proposition 4.1 If $H$ and $K$ are separable infinite dimensional Hilbert spaces, and $A \in B(H, K)$ is a compact contraction, then $X(A)$ is $2^{6}$-completely isomorphic to $X^{d}(\mathbf{D}(A))$. 
Proof Let $\alpha=\left(\alpha_{i}\right)=\mathbf{D}(A)$. By Lemma 3.2(1), we can assume that $A=|A|=$ $\left(A^{*} A\right)^{1 / 2}$, and $H=K$. If $A$ has finite rank, (4-1) shows that $X(A)$ is completely isometric to $X^{d}(\alpha)$. Otherwise, write $A=\sum_{i} \alpha_{i} \xi_{i} \otimes \xi_{i}$, for some orthonormal system $\left(\xi_{i}\right)_{i=1}^{\infty}$ in $H$. Let $P$ be the orthogonal projection onto $K=\operatorname{span}\left[\xi_{i}: i \in \mathbb{N}\right]$. Set $Q=I-P$, and $L=Q(H)$. By Lemma 3.2(2), $X(A)$ is $\sqrt{2}$-completely isomorphic to $X(K, K, P A P) \oplus X(L, L, Q A Q)$. Furthermore, $Q A Q=0$, hence $X(L, L, Q A Q)=L_{\mathbf{R}}$. It is easy to see that $X(K, K, P A P)=X^{d}(\alpha)$. By Proposition 3.7, the latter space is $4 \sqrt{2}$-completely isomorphic to $X^{d}(\alpha) \oplus \mathbf{R}$. Thus,

$$
\begin{aligned}
X(A) & \stackrel{\sqrt{2}}{\simeq} X(K, K, P A P) \oplus X(L, L, Q A Q) \stackrel{4 \sqrt{2}}{\simeq} X^{d}(\alpha) \oplus \mathbf{R} \oplus L_{\mathbf{R}} \\
& \stackrel{\sqrt{2}}{\simeq} X^{d}(\alpha) \oplus\left(\ell_{2} \oplus L\right)_{\mathbf{R}}=X^{d}(\alpha) \oplus \mathbf{R} \stackrel{4 \sqrt{2}}{\simeq} X^{d}(\alpha) .
\end{aligned}
$$

We will also use a related observation.

Lemma 4.2 For every compact contraction $A \in B\left(\ell_{2}\right)$, and every $\varepsilon>0$, there exists $\alpha \in c_{0}$ such that $X(A) \stackrel{1+\varepsilon}{\simeq} X^{d}(\alpha)$.

Proof Indeed, let $\beta=\mathbf{D}(A)$. If $A$ is finite rank, then $X(A)$ is completely isometric to $X^{d}(\beta)$. If $\operatorname{rank} A=\infty$, assume (by Lemma 3.2) that $A=|A|$. Then $\beta_{1} \geq \beta_{2} \geq$ $\ldots>0$ is the list of all positive eigenvalues of $A$. Denote the corresponding norm 1 eigenvectors by $\xi_{i}$. Let $H=\operatorname{span}\left[\xi_{i}: i \in \mathbb{N}\right]$, and $K=\operatorname{ker} A$. Clearly, $K$ and $H$ are mutually orthogonal subspaces of $\ell_{2}$. Let $\left(\eta_{j}\right)_{j \in J}$ be the orthogonal basis of $K$. Find a sequence $\left(\gamma_{j}\right)_{j \in J}$ of positive numbers, satisfying $\sum_{j \in J} \gamma_{i}^{2}<\varepsilon^{2}$. Consider the compact contraction $\tilde{A} \in B\left(\ell_{2}\right)$, defined by $\tilde{A} \xi_{i}=\beta_{i} \xi_{i}$, and $\tilde{A} \eta_{j}=\gamma_{j} \eta_{j}$. Then $\|A-\tilde{A}\|_{2}<\varepsilon$. By (4-1), the formal identity map id $: X(\tilde{A}) \rightarrow X(A)$ is a complete contraction, and $\left\|i d^{-1}\right\|_{c b}<1+\varepsilon$. We complete the proof by identifying $X(\tilde{A})$ with the space $X^{d}(\alpha)$, where the sequence $\alpha=\left(\alpha_{i}\right)$ is the "join" of the sequences $\beta$ and $\gamma$ (that is, any number $c \in[0,1]$ occurs in $\alpha$ as many times as it occurs in the sequences $\beta$ and $\gamma$ combined).

Now denote by $\mathcal{S}$ the set of all sequences $\left(\alpha_{i}\right)_{i \in \mathbb{N}}$ satisfying $1 \geq \alpha_{1} \geq \alpha_{2} \geq \ldots \geq 0$, and $\lim _{i} \alpha_{i}=0$. The rest of this section is devoted to the spaces $X^{d}(\alpha)(\alpha \in \mathcal{S})$. We translate the relations between sequences $\alpha, \beta \in \mathcal{S}$ to relations between the corresponding spaces $X^{d}(\alpha)$ and $X^{d}(\beta)$. We say that the sequence $\alpha$ dominates $\beta$ $(\alpha \succ \beta)$ if there exists a set $S \subset \mathbb{N})$ and $K>0$ s.t. $\sum_{i \in S} \beta_{i}^{2}<\infty$, and $K \alpha_{i} \geq \beta_{i}$ for any $i \notin S$. We say $\alpha$ is equivalent to $\beta(\alpha \sim \beta)$ if $\alpha \succ \beta$, and $\beta \succ \alpha$.

Clearly, the relation $\succ$ is reflexive and transitive. The relation $\sim$ is, in addition to this, symmetric. For instance, to establish the transitivity of $\succ$, suppose $\alpha \succ \beta$, and $\beta \succ \gamma$, 
and show that $\alpha \succ \gamma$. Note that there exist sets $S_{1}$ and $S_{2}$, and constants $K_{1}$ and $K_{2}$, s.t. $\beta_{i} \leqslant K_{1} \alpha_{i}$ for $i \notin S_{1}, \gamma_{i} \leqslant K_{2} \beta_{i}$ for $i \notin S_{2}, \sum_{i \in S_{1}} \beta_{i}^{2}<\infty$, and $\sum_{i \in S_{2}} \gamma_{i}^{2}<\infty$. Let $S=S_{1} \cup S_{2}$, and $K=K_{1} K_{2}$. Then $\gamma_{i} \leqslant K \alpha_{i}$ for $i \notin S$. Moreover,

$$
\sum_{i \in S} \gamma_{i}^{2}=\sum_{i \in S_{2}} \gamma_{i}^{2}+\sum_{i \in S_{1} \backslash S_{2}} \gamma_{i}^{2} \leqslant \sum_{i \in S_{2}} \gamma_{i}^{2}+\sum_{i \in S_{1}} K_{2}^{2} \beta_{i}^{2}<\infty,
$$

which is what we need. The other properties are proved in a similar fashion.

Proposition 4.3 For $\alpha, \beta \in \mathcal{S}, \alpha \sim \beta$ if and only if there exists a set $S \subset \mathbb{N}$ and a constant $K$ s.t. $\sum_{i \in S}\left(\alpha_{i}^{2}+\beta_{i}^{2}\right)<\infty$, and $K^{-1} \alpha_{i} \leqslant \beta_{i} \leqslant K \alpha_{i}$ for $i \notin S$.

Proof If $S$ and $K$ with the properties described above exist, then they witness the fact that $\alpha \prec \beta$ and $\alpha \succ \beta$, hence $\alpha \sim \beta$. Conversely, suppose $\alpha \prec \beta$ and $\alpha \succ \beta$. Then there exist a constant $K$, and sets $S_{1}$ and $S_{2}$, s.t. $\alpha_{i} \leqslant K \beta_{i}$ for $i \notin S_{1}, \beta_{i} \leqslant K \alpha_{i}$ for $i \notin S_{2}, \sum_{i \in S_{1}} \alpha_{i}^{2}<\infty$, and $\sum_{i \in S_{2}} \beta_{i}^{2}<\infty$. By reducing $S_{1}$ and $S_{2}$ further, we can assume that $\alpha_{i}>K \beta_{i}$ for each $i \in S_{1}$, and $\beta_{i}>K \alpha_{i}$ for each $i \in S_{2}$. Then $\sum_{i \in S_{1}} \beta_{i}^{2}<\infty$, and $\sum_{i \in S_{2}} \alpha_{i}^{2}<\infty$. Therefore, $S=S_{1} \cup S_{2}$ has the required properties.

The main result of this section is:

Theorem 4.4 For $\alpha, \beta \in \mathcal{S}, X^{d}(\alpha)$ embeds completely isomorphically into $X^{d}(\beta)$ if and only if $\alpha \prec \beta$.

From this we immediately obtain

Corollary 4.5 Suppose $\alpha, \beta \in \mathcal{S}$. The following three statements are equivalent.

(1) $X^{d}(\alpha)$ is completely isomorphic to $X^{d}(\beta)$.

(2) $X^{d}(\alpha)$ embeds completely isomorphically into $X^{d}(\beta)$, and vice versa.

(3) $\alpha \sim \beta$.

Proof $(1) \Rightarrow(2)$ is trivial, while $(2) \Rightarrow$ (3) follows from Theorem 4.4 and Proposition 4.3. To establish (3) $\Rightarrow$ (1), suppose $\alpha \sim \beta$. By Proposition 4.3, there exists a set $\mathcal{I}$ and $K>0$ s.t. $\sum_{i \in \mathcal{I}}\left(\alpha_{i}^{2}+\beta_{i}^{2}\right)<\infty$, and $K^{-1} \alpha_{i} \leqslant \beta_{i} \leqslant K \alpha_{i}$ for any $i \notin \mathcal{I}$. By Corollary 3.5, the spaces $E_{\alpha}=\operatorname{span}\left[e_{i}: i \in \mathcal{I}\right] \hookrightarrow X^{d}(\alpha)$ and $E_{\beta}=\operatorname{span}\left[e_{i}: i \in\right.$ $\mathcal{I}] \hookrightarrow X^{d}(\beta)$ are completely isomorphic to $\mathbf{R}$. By (4-2), the formal identity map from $F_{\alpha}=\operatorname{span}\left[e_{i}: i \notin \mathcal{I}\right] \hookrightarrow X^{d}(\alpha)$ to $F_{\beta}=\operatorname{span}\left[e_{i}: i \notin \mathcal{I}\right] \hookrightarrow X^{d}(\beta)$ is a complete isomorphism. By Lemma 3.2(2), $X^{d}(\alpha) \simeq E_{\alpha} \oplus F_{\alpha}$, and $X^{d}(\beta) \simeq E_{\beta} \oplus F_{\beta}$. Therefore, the formal identity map from $X^{d}(\alpha)$ to $X^{d}(\beta)$ is a complete isomorphism. 
The proof of Theorem 4.4 follows from the next two lemmas.

Lemma 4.6 If $\alpha, \beta \in \mathcal{S}$ and $\beta \prec \alpha$, then $X^{d}(\beta)$ embeds completely isomorphically into $X^{d}(\alpha)$.

Proof By Corollary 3.8, $X^{d}(\alpha)$ is completely isomorphic to $X(A)$, where $A \in B\left(\ell_{2} \oplus_{2}\right.$ $\left.\ell_{2}\right)$ is defined by $A=\operatorname{diag}(\alpha) \oplus 0$. Let $\left(\xi_{i}\right)$ and $\left(\xi_{i}^{\prime}\right)$ be orthonormal bases in the first and second copies of $\ell_{2}$, respectively. Then $X(A)$ is the closed linear span of the vectors $\xi_{i} \oplus \alpha_{i} \xi_{i}$ and $\xi_{i}^{\prime} \oplus 0(i \in \mathbb{N})$. Find a sequence $\phi_{i} \in[0, \pi / 2]$ s.t. $\beta_{i}=\cos \phi_{i} \cdot \alpha_{i}$. Define an orthonormal system $\eta_{i}=\cos \phi_{i} \xi_{i}+\sin \phi_{i} \xi_{i}^{\prime}$. For $i \in \mathbb{N}$ consider

$$
f_{i}=\eta_{i} \oplus \beta_{i} \xi_{i}=\cos \phi_{i}\left(\xi_{i} \oplus \alpha_{i} \xi_{i}\right)+\sin \phi_{i}\left(\xi_{i}^{\prime} \oplus 0\right) .
$$

Then $f_{i} \in X(A)$, and $\operatorname{span}\left[f_{i}: i \in \mathbb{N}\right]=X^{d}(\beta)$.

Lemma 4.7 Suppose $\alpha, \beta \in \mathcal{S}, E$ is a subspace of $X^{d}(\alpha)$, and a completely bounded map $U: E \rightarrow X^{d}(\beta)$ has bounded inverse (in the terminology of [18], $E$ is completely semi-isomorphic to $\left.X^{d}(\beta)\right)$. Then $\beta \prec \alpha$.

Proof We rely heavily on Wielandt's Minimax Theorem ([2, Theorem III.6.5]): if $c_{1} \geq c_{2} \geq \ldots \geq 0$ are eigenvalues of positive compact operator $T$, then, for any finite increasing sequence $i_{1}<\ldots<i_{k}$ of positive integers,

$$
\sum_{j=1}^{k} c_{i_{j}}=\sup _{E_{1} \hookrightarrow \ldots \hookrightarrow E_{k}} \min _{x_{j} \in E_{j},\left(x_{j}\right) \text { orthonormal }}\left\langle T x_{j}, x_{j}\right\rangle,
$$

where the supremum is taken over all subspaces $E_{1} \hookrightarrow \ldots \hookrightarrow E_{k}$ of the domain of $T$, with $\operatorname{dim} E_{j}=i_{j}$ for $1 \leqslant j \leqslant k$. Actually, the theorem is stated in [2] for operators on finite dimensional spaces, but a generalization to compact operators is easy to obtain. Applying the above identity to $T=S^{*} S$, where $S$ is a compact operator with singular numbers $s_{1} \geq s_{2} \geq \ldots \geq 0$, we obtain:

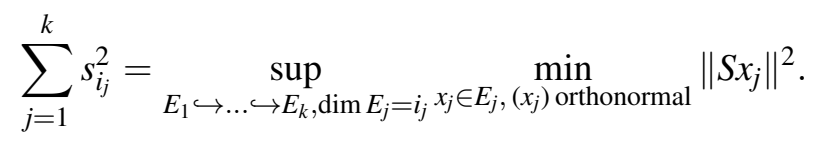

In our situation, assume $\|U\|_{c b}=1$. Let $c=\left\|U^{-1}\right\|, A=\operatorname{diag}\left(\alpha_{i}\right), B=\operatorname{diag}\left(\beta_{i}\right)$, $B^{\prime}=B U$. Denote the singular values of $B^{\prime}$ by $\left(\beta_{i}^{\prime}\right)$. Clearly, $\beta_{i}^{\prime} \leqslant \beta_{i} \leqslant c \beta_{i}^{\prime}$ for every $i \in \mathbb{N}$. 
Pick an orthonormal system $\left(x_{j}\right)_{j=1}^{k}$ in $E$ (the domain of $U$ ). Let $u$ be the formal identity from $\mathbf{R}^{k}=\left(\ell_{2}^{k}\right)_{\mathbf{R}}$ (the $k$-dimensional row space) to $\operatorname{span}\left[x_{j}: 1 \leqslant j \leqslant k\right]$. Then (compare with the proof of Lemma 3.3)

$$
\|u\|_{c b}^{2}=\max \left\{1,\|A u\|_{2}^{2}\right\} \leqslant 1+\sum_{j=1}^{k}\left\|A x_{j}\right\|^{2},
$$

and (since $U$ is a complete contraction)

$$
\|u\|_{c b}^{2} \geq\|U u\|_{c b}^{2} \geq\|B U u\|_{2}^{2}=\sum_{j=1}^{k}\left\|B^{\prime} x_{j}\right\|^{2} .
$$

Thus, $\sum_{j=1}^{k}\left\|B^{\prime} x_{j}\right\|^{2} \leqslant \sum_{j=1}^{k}\left\|A x_{j}\right\|^{2}+1$ for any orthonormal family $\left(x_{j}\right)_{j=1}^{k}$. By (4-3),

$$
1+\sum_{j=1}^{k} \alpha_{i_{j}}^{2} \geq \sum_{j=1}^{k} \beta_{i_{j}}^{\prime 2}
$$

for any $i_{1}<\ldots<i_{k}$ (indeed, when computing $\sum_{j=1}^{k} \alpha_{i_{j}}^{2}$, we are taking the supremum over a larger family of subspaces $\left(E_{j}\right)$, than when we are computing $\sum_{j=1}^{k} \beta_{i_{j}}^{\prime 2}$ ).

Now let $\mathcal{I}=\left\{i \in \mathbb{N}: \beta_{i}^{\prime}>2 \alpha_{i}\right\}$. Then $\sum_{i \in \mathcal{I}} \beta_{i}^{\prime 2} \leqslant 2$. Indeed, otherwise there exists a sequence $i_{1}<\ldots<i_{k}$ of elements of $\mathcal{I}$ s.t. $C=\sum_{j=1}^{k} \beta_{i_{j}}^{\prime 2}>2$. Then, by (4-4), $\sum_{j=1}^{k} \alpha_{i_{j}}^{2} \geq C-1$. On the other hand, $\sum_{j=1}^{k} \alpha_{i_{j}}^{2} \leqslant C / 2$, a contradiction.

Developing the ideas of this proof, we obtain:

Theorem 4.8 Suppose $\alpha, \beta \in \mathcal{S}$, and there exists an isomorphism $U: X^{d}(\alpha) \rightarrow$ $X^{d}(\beta)$ with $\|U\|_{c b},\left\|U^{-1}\right\|_{c b} \leqslant C$. Then the formal identity map id: $X^{d}(\alpha) \rightarrow X^{d}(\beta)$ satisfies $\|i d\|_{c b},\left\|i d^{-1}\right\|_{c b}<4 C^{2}$.

Proof As in the proof of Lemma 4.7, let $A=\operatorname{diag}\left(\alpha_{i}\right), B=\operatorname{diag}\left(\beta_{i}\right)$, and $B^{\prime}=B U$. By Lemma 3.3, $\left\|B^{\prime} u\right\|_{2} \leqslant C \max \left\{\|A u\|_{2},\|u\|\right\}$ for any $u: \ell_{2} \rightarrow X^{d}(\alpha)$. Denote the singular numbers of $B^{\prime}$ by $\left(\beta_{i}^{\prime}\right)$, and note that $\beta_{i} / C \leqslant \beta_{i}^{\prime} \leqslant C \beta_{i}$ for every $i$. Reasoning as in the proof of Lemma 4.7, we see that

$$
C^{2}\left(1+\sum_{j=1}^{k} \alpha_{i_{j}}^{2}\right) \geq \sum_{j=1}^{k} \beta_{i_{j}}^{\prime 2}
$$

Let $\mathcal{I}=\left\{i: \beta_{i}^{\prime}>2 C \alpha_{i}\right\}$. As in the preceding proof, $\sum_{i \in \mathcal{I}} \beta_{i}^{\prime 2}<2 C^{2}$. Therefore, $\sum_{i \in \mathcal{I}} \beta_{i}^{2}<2 C^{4}$, and $\beta_{i} \leqslant 2 C^{2} \alpha_{i}$ for $i \notin \mathcal{I}$. 
Next we show that $\left\|i d: X^{d}(\alpha) \rightarrow X^{d}(\beta)\right\|_{c b}<4 C^{2}$. As before, denote the canonical bases in $X^{d}(\alpha)$ and $X^{d}(\beta)$ by $\left(e_{i}(\alpha)\right)_{i \in \mathbb{N}}$ and $\left(e_{i}(\beta)\right)_{i \in \mathbb{N}}$, respectively. By (4-2), $Y_{1}=\operatorname{span}\left[e_{i}(\alpha): i \in \mathcal{I}\right]$ and $Y_{0}=\operatorname{span}\left[e_{i}(\alpha): i \notin \mathcal{I}\right]$ are completely contractively complemented subspaces of $X^{d}(\alpha)$. Moreover,

$$
\begin{aligned}
\left\|\left.i d\right|_{Y_{1}}\right\|_{c b} \leqslant\left\|V:\left(Y_{1}\right)_{\mathbf{R}} \rightarrow X^{d}(\beta)\right\|_{c b} & =\max \left\{1,\|B V\|_{c b}\right\} \\
& \leqslant \max \left\{1,\left(\sum_{i \in \mathcal{I}} \beta_{i}^{2}\right)^{1 / 2}\right\}<2 C^{2}
\end{aligned}
$$

(here, $V$ is the formal identity from $\left(Y_{1}\right)_{\mathbf{R}}$ to $\left.\operatorname{span}\left[e_{i}(\beta): i \in \mathcal{I}\right]\right)$. By Lemma 3.3 and (4-2), $\left\|\left.i d\right|_{Y_{0}}\right\|_{c b}<2 C^{2}$. Therefore,

$$
\left\|i d: X^{d}(\alpha) \rightarrow X^{d}(\beta)\right\|_{c b} \leqslant\left\|\left.i d\right|_{Y_{1}}\right\|_{c b}+\left\|\left.i d\right|_{Y_{0}}\right\|_{c b}<4 C^{2} .
$$

The norm of $i d: X^{d}(\beta) \rightarrow X^{d}(\alpha)$ is computed the same way.

\section{Completely unconditional bases}

In this section, we further investigate bases in spaces $X(A)$, where $A \in B(H, K)$ is a compact contraction. A subspace $E$ of $X(A)$ is isometric to $X\left(\left.A\right|_{E}\right)$. As $\left.A\right|_{E}$ is a compact contraction, (4-1) implies that $E$ has a 1-completely unconditional basis. The key result of this section is Proposition 5.2, establishing the uniqueness of a completely unconditional basis in $E$ (the existence of such a basis has been established by Proposition 4.1). We also show that the canonical basis (and therefore, every completely unconditional basis) in a completely complemented subspace of $X^{d}(\alpha)$ is equivalent to a subsequence of the canonical basis of $X^{d}(\alpha)$. Moreover, there exists $\alpha \in \mathcal{S}$ such that the canonical basis in every complemented subspace of $X^{d}(\alpha)$ is equivalent to a subsequence of the canonical basis of $X^{d}(\alpha)$ (Theorem 5.6). In general, the last statement need not be true (Remark 5.5).

First we show that any completely unconditional basic sequence in an $X(A)$ space corresponds to a canonical basis of $X^{d}(\beta)$, for some $\beta$.

Proposition 5.1 Suppose $\mathcal{I}$ is a finite or countable set, and $\left(e_{i}^{\prime}\right)_{i \in \mathcal{I}}$ is a a $C$-completely unconditional basic sequence in $X(A)\left(A \in B\left(\ell_{2}\right)\right.$ is a contraction, not necessarily compact). Let $Y=\operatorname{span}\left[e_{i}^{\prime}: i \in \mathcal{I}\right]$, and define the sequence $\beta=\left(\beta_{i}\right)$ by setting $\beta_{i}=\left\|A e_{i}^{\prime}\right\|$. Consider the operator $T: Y \rightarrow X^{d}(\beta): e_{i}^{\prime} \mapsto e_{i}$, where $\left(e_{i}\right)$ is the canonical basis of $X^{d}(\beta)$. Then $\|T\|_{c b} \leqslant C$ and $\left\|T^{-1}\right\|_{c b} \leqslant C^{2}$. 
Proof As noted in Section 2,

$$
C^{-2}\left\|\sum \alpha_{i} e_{i}^{\prime}\right\|^{2} \leqslant \sum\left|\alpha_{i}\right|^{2} \leqslant C^{2}\left\|\sum \alpha_{i} e_{i}^{\prime}\right\|^{2}
$$

for any finite sequence of scalars $\left(\alpha_{i}\right)$. Thus, $\|T\|,\left\|T^{-1}\right\| \leqslant C$.

Let $B=\operatorname{diag}(\beta)$ (note that $B=B^{*}$ ). By Lemma 3.3, it suffices to show that

$$
\|B T u\|_{2} \leqslant C \max \left\{\|A u\|_{2},\|u\|\right\}
$$

for any $u: \ell_{2} \rightarrow Y=X\left(\left.A\right|_{Y}\right)$, and

$$
\left\|A T^{-1} u\right\|_{2} \leqslant C^{2} \max \left\{\|B u\|_{2},\|u\|\right\}
$$

for any $u: \ell_{2} \rightarrow X^{d}(\beta)$. By Lemma 3.3, the complete unconditionality of $\left(e_{i}^{\prime}\right)$ implies:

$$
\begin{aligned}
& \|A \Lambda u\|_{2} \leqslant C \max \left\{\|A u\|_{2},\|u\|\right\}, \\
& \|A u\|_{2} \leqslant C \max \left\{\|A \Lambda u\|_{2},\|u\|\right\}
\end{aligned}
$$

whenever $\Lambda=\operatorname{diag}\left(\lambda_{i}\right)$ (that is, $\Lambda e_{i}^{\prime}=\lambda_{i} e_{i}^{\prime}$ ), with $\lambda_{i}= \pm 1$ for each $i$, and $u \in$ $B\left(\ell_{2}, Y\right)$. Note that $\|A u\|_{2}^{2}=\operatorname{tr}\left(A^{*} A v\right)$, where $v=u u^{*}$. Therefore, (5-4) is equivalent to

$$
\begin{array}{ll}
\operatorname{tr}\left(\Lambda^{*} A^{*} A \Lambda v\right) & \leqslant C^{2} \max \left\{\operatorname{tr}\left(A^{*} A v\right), 1\right\}, \\
\operatorname{tr}\left(A^{*} A v\right) & \leqslant C^{2} \max \left\{\operatorname{tr}\left(\Lambda^{*} A^{*} A \Lambda v\right), 1\right\}
\end{array}
$$

whenever $v \geq 0$ and $\|v\|=1$. But $\left\langle\Lambda^{*} A^{*} A \Lambda e_{i}^{\prime}, e_{j}^{\prime}\right\rangle=\lambda_{i} \overline{\lambda_{j}}\left\langle A e_{i}^{\prime}, A e_{j}^{\prime}\right\rangle$. Averaging over $\lambda_{i}= \pm 1$ for each $i$, we see that $\operatorname{Ave}_{\Lambda} \Lambda^{*} A^{*} A \Lambda=T^{*} B^{2} T$ (since $\left\langle T^{*} B^{2} T e_{i}^{\prime}, e_{j}^{\prime}\right\rangle=$ $\delta_{i j}\left\langle A e_{i}^{\prime}, A e_{j}^{\prime}\right\rangle$, where $\delta_{i j}$ is Kronecker's delta). Therefore, by (5-5),

$$
\begin{aligned}
\operatorname{tr}\left(T^{*} B^{2} T v\right) & =\operatorname{Ave}_{\Lambda} \operatorname{tr}\left(\Lambda^{*} A^{*} A \Lambda v\right) \\
& \leqslant \sup _{\Lambda} \operatorname{tr}\left(\Lambda^{*} A^{*} A \Lambda v\right) \leqslant C^{2} \max \left\{\operatorname{tr}\left(A^{*} A v\right), 1\right\}
\end{aligned}
$$

whenever $v$ is a positive contraction. Thus, for any contraction $u$,

$$
\begin{aligned}
\|B T u\|_{2}^{2} & =\operatorname{tr}\left(T^{*} B^{2} T u u^{*}\right) \\
& \leqslant C^{2} \max \left\{\operatorname{tr}\left(A^{*} A u u^{*}\right), 1\right\}=C^{2} \max \left\{\|A u\|_{2},\|u\|\right\}^{2},
\end{aligned}
$$

which proves (5-2).

To establish (5-3), we show that, for every $w: \ell_{2} \rightarrow Y$, we have

$$
\|A w\|_{2} \leqslant C \max \left\{\|B T w\|_{2},\|w\|\right\}
$$

Indeed, let $w=T^{-1} u$. Then, by (5-6) and (5-1),

$$
\begin{aligned}
\left\|A T^{-1} u\right\|_{2} & =\|A w\|_{2} \leqslant C \max \left\{\|B T w\|_{2},\|w\|\right\} \\
& \leqslant C \max \left\{\|B u\|_{2}, C\|u\|\right\} \leqslant C^{2} \max \left\{\|B u\|_{2},\|u\|\right\} .
\end{aligned}
$$


Moreover, (5-6) is equivalent to the following: for any non-negative, norm one $v \in$ $B\left(X^{d}(\alpha)\right)$, we have

$$
C^{2} \max \left\{\operatorname{tr}\left(T^{*} B^{2} T v\right), 1\right\} \geq \operatorname{tr}\left(A^{*} A v\right) .
$$

As we have established before, $\operatorname{tr}\left(T^{*} B^{2} T v\right)=\operatorname{Ave}_{\Lambda} \operatorname{tr}\left(\Lambda^{*} A^{*} A \Lambda v\right)$. By the linearity and positivity of the trace,

$$
0 \leqslant \inf _{\Lambda} \operatorname{tr}\left(\Lambda^{*} A^{*} A \Lambda v\right) \leqslant \operatorname{tr}\left(T^{*} B^{2} T v\right) \leqslant \sup _{\Lambda} \operatorname{tr}\left(\Lambda^{*} A^{*} A \Lambda v\right) .
$$

Thus, for some $\Lambda, \operatorname{tr}\left(\Lambda^{*} A^{*} A \Lambda v\right) \leqslant \operatorname{tr}\left(T^{*} B^{2} T v\right)$. By (5-5),

$$
\operatorname{tr}\left(A^{*} A v\right) \leqslant C^{2} \max \left\{\operatorname{tr}\left(\Lambda^{*} A^{*} A \Lambda v\right), 1\right\} \leqslant C^{2} \max \left\{\operatorname{tr}\left(T^{*} B^{2} T v\right), 1\right\},
$$

which implies (5-7).

Proposition 5.2 For $\alpha \in c_{0}$, the completely unconditional basis in $X^{d}(\alpha)$ is unique (up to permutative equivalence). More precisely: if $\left(g_{i}\right)$ is a $C$-completely unconditional basis in $X^{d}(\alpha)$, then it is $16 C^{11}$-equivalent (up to a permutation) to the canonical basis in $X^{d}(\alpha)$.

Proof Let $\left(e_{i}(\alpha)\right)$ be the canonical basis of $X^{d}(\alpha)$. Set $\beta_{i}=\left\|A g_{i}\right\|$, and let $\left(e_{i}(\beta)\right)$ the canonical basis of $X^{d}(\beta)$. By Proposition 5.1, the map $T: X^{d}(\alpha) \rightarrow X^{d}(\beta): g_{i} \mapsto e_{i}(\beta)$ satisfies $\|T\|_{c b} \leqslant C,\left\|T^{-1}\right\|_{c b} \leqslant C^{2}$. By Theorem 4.8, id : $X^{d}(\beta) \rightarrow X^{d}(\alpha)$ satisfy $\|i d\|_{c b},\left\|i d^{-1}\right\|_{c b}<4 C^{4}$. Thus, the operator $U=i d \circ T$ is a complete isomorphism on $X^{d}(\alpha)$, with $U g_{i}=e_{i},\|U\|_{c b}<4 C^{5}$, and $\left\|U^{-1}\right\|_{c b}<4 C^{6}$.

Corollary 5.3 Suppose $\alpha \in \mathcal{S}$, and $Y$ is a $C$-completely complemented subspace of $X^{d}(\alpha)$. Then $Y$ is $2^{6} C^{2}$-completely isomorphic to $X^{d}\left(\alpha^{\prime}\right)$, where $\alpha^{\prime}$ is a subsequence of $\alpha$.

Proof Let $P$ be a projection from $X^{d}(\alpha)$ onto $Y$, with $\|P\|_{c b} \leqslant C$. Then $X^{d}(\alpha)$ is $2 C$-completely isomorphic to $Y \oplus Z$, where $Z=\operatorname{ker} P$. By Lemma 4.2, $Y$ and $Z$ are $\sqrt{2}$-completely isomorphic to $X^{d}(\beta)$ and $X^{d}\left(\beta^{\prime}\right)$, respectively, where $\beta$ and $\beta^{\prime}$ belong to $\mathcal{S}$. By Lemma 3.2(2), $X^{d}(\alpha)$ is $4 C$-completely isomorphic to $X^{d}(\gamma)$, where $\gamma=\left(\gamma_{i}\right) \in \mathcal{S}$ is the "join" of $\beta=\left(\beta_{i}\right)$ and $\beta^{\prime}=\left(\beta_{i}^{\prime}\right)$. More precisely, the sequence $\gamma$ has the property that, for every $c \in[0,1]$,

$$
\left|\left\{i: \gamma_{i}=c\right\}\right|=\left|\left\{i: \beta_{i}=c\right\}\right|+\left|\left\{i: \beta_{i}^{\prime}=c\right\}\right| .
$$

Denoting the canonical basis of $X^{d}(\gamma)$ by $\left(e_{i}(\gamma)\right)$, we see that $X^{d}(\beta)=\operatorname{span}\left[e_{i}(\gamma)\right.$ : $i \in I]$, for some infinite set $I \subset \mathbb{N}$. By Theorem 4.8, the formal identity id : $X^{d}(\gamma) \rightarrow X^{d}(\alpha): e_{i}(\gamma) \mapsto e_{i}(\alpha)$ satisfies $\|i d\|_{c b},\left\|i d^{-1}\right\|_{c b}<8 C$. In particular, $X^{d}(\beta)$ $2^{6} C^{2}$-completely isomorphic to $\operatorname{span}\left[e_{i}(\alpha): i \in I\right]$. 
Remark 5.4 By [17], the completely unconditional basis in $\mathbf{R} \oplus \mathbf{C}$ is unique up to a permutation.

Remark 5.5 In general, the canonical basis of a subspace of $X^{d}(\alpha)(\alpha \in \mathcal{S})$ need not be equivalent to a subsequence of the canonical basis of $X^{d}(\alpha)$. For instance, suppose the sequence $\alpha=\left(\alpha_{i}\right)$ and $\beta=\left(\beta_{i}\right)$ are defined by setting $\alpha_{i}=2^{-n^{2}}, \beta_{i}=2^{-n^{2}-n}$ for $4^{n^{2}} \leqslant i<4^{(n+1)^{2}}(n \in\{0\} \cup \mathbb{N})$. By Lemma 4.6, $X^{d}(\beta)$ embeds completely isomorphically into $X^{d}(\alpha)$. However, $\left(e_{i}(\beta)\right)$ (the canonical basis of $X^{d}(\beta)$ ) is not equivalent to any subsequence of the canonical basis $\left(e_{i}(\alpha)\right)$ of $X^{d}(\alpha)$. Indeed, suppose, for the sake of achieving a contradiction, that there exists a complete isomorphism $T$ from $X^{d}(\beta)$ to a subspace of $X^{d}(\alpha)$, mapping $e_{i}(\beta)$ to $e_{k_{i}}(\alpha)$. Fix $n \in \mathbb{N}$ with $\max \left\{\|T\|_{c b},\left\|T^{-1}\right\|_{c b}\right\}<2^{n / 2}$. Consider the sets

$$
\begin{aligned}
& I_{n}=\left\{4^{n^{2}} \leqslant i<4^{(n+1)^{2}}: k_{i}<4^{(n+1)^{2}}\right\}, \\
& J_{n}=\left\{4^{n^{2}} \leqslant i<4^{(n+1)^{2}}: k_{i} \geq 4^{(n+1)^{2}}\right\} .
\end{aligned}
$$

By Pigeon-Hole Principle, with $I_{n}$ or $J_{n}$ has the cardinality grater than $4^{n^{2}+2 n}$. If $\left|I_{n}\right|>4^{n^{2}+2 n}$, consider

$$
x=\sum_{i \in I_{n}} E_{i 1} \otimes e_{i}(\beta) \in M_{4(n+1)^{2}}\left(X^{d}(\beta)\right)
$$

(recall that $E_{i 1}$ is the "matrix unit" with 1 on the intersection of the first column and the $i$-th row, and zeroes everywhere else). By (4-2),

$$
\|x\|_{M_{4^{(n+1)^{2}}}^{2}\left(X^{d}(\beta)\right)}^{2}=\max \left\{1,\left|I_{n}\right| \cdot 2^{-n^{2}-n}\right\}=\left|I_{n}\right| \cdot 2^{-n^{2}-n} .
$$

However, by (4-2) again,

$$
\left\|\left(I_{M_{4^{(n+1)^{2}}}} \otimes T\right) x\right\|_{M_{4^{(n+1)^{2}}}\left(X^{d}(\alpha)\right)}^{2} \geq \max \left\{1,\left|I_{n}\right| \cdot 2^{-n^{2}-n}\right\}=\left|I_{n}\right| \cdot 2^{-n^{2}},
$$

yielding $\|T\|_{c b}^{2} \geq 2^{-n}$. If $J_{n}>4^{n^{2}+2 n}$, consider

$$
x=\sum_{i \in J_{n}} E_{i 1} \otimes e_{i}(\beta) \in M_{4^{(n+1)^{2}}}\left(X^{d}(\beta)\right) .
$$

As before, $\|x\|^{2}=\left|J_{n}\right| \cdot 2^{-n^{2}-n}$, while

$$
\left\|\left(I_{M_{4^{(n+1)^{2}}}} \otimes T\right) x\right\|_{M_{4^{(n+1)^{2}}}\left(X^{d}(\alpha)\right)}^{2} \geq \max \left\{1,\left|I_{n}\right| \cdot 2^{-(n+1)^{2}}\right\}=\left|J_{n}\right| \cdot 2^{-(n+1)^{2}},
$$

hence $\left\|T^{-1}\right\|_{c b}^{2} \geq 2^{n+1}$. Thus, $\max \left\{\|T\|_{c b},\left\|T^{-1}\right\|_{c b}\right\} \geq 2^{n / 2}$, which yields the desired contradiction.

In certain situations, the canonical basis for every subspace of $X^{d}(\alpha)$ is equivalent to a subsequence of the canonical basis of $X^{d}(\alpha)$. 
Theorem 5.6 For any $a>1$ there exists $\alpha \in \mathcal{S} \backslash \ell_{2}$ such that any subspace $Y$ of $X^{d}(\alpha)$ (finite or infinite dimensional) has a 1 -completely unconditional basis, a-equivalent to a subsequence of the canonical basis of $X^{d}(\alpha)$.

Combining this result with Proposition 5.2, we obtain

Corollary 5.7 There exists $\alpha \in \mathcal{S} \backslash \ell_{2}$ such that any $C$-completely unconditional basic sequence in $X^{d}(\alpha)$ is $A C^{B}$-equivalent to a subsequence of the canonical basis of $X^{d}(\alpha)$ (here, $A$ and $B$ are positive).

Proof of Theorem 5.6 Assume $a<2$. Pick a sequence of integers $1=N_{0}<N_{1}<$ ..., s.t. $N_{k}>2 N_{k-1}$ for each $k$. Define a sequence $\alpha=\left(\alpha_{i}\right)$ by setting $\alpha_{2 i}=a^{-k}$ for $N_{k} \leqslant i<N_{k+1}, \alpha_{2 i-1}=0$ for any $i \in \mathbb{N}$. Clearly, $\alpha \in c_{0} \backslash \ell_{2}$. Let $A=\operatorname{diag}(\alpha)$. For a subspace $Y$ of $X(A)$, let $\beta=\left(\beta_{i}\right)$ is the sequence of singular values of $\left.A\right|_{Y}$. Define the set $\mathcal{I}_{1}$ by setting $\mathcal{I}_{1}=\{1, \ldots, M\}$ if $\operatorname{rank}\left(\left.A\right|_{Y}\right)=M<\infty$, and $\mathcal{I}_{1}=\mathbb{N}$ if $\operatorname{rank}\left(\left.A\right|_{Y}\right)=\infty$. We can also assume that the elements of $\left(\beta_{i}\right)_{i \in \mathcal{I}_{1}}$ are listed in the non-increasing order. Then $\beta_{i} \leqslant \alpha_{2 i}$ for each $i$.

Denote the normalized eigenvectors of $\left.\left(\left.A\right|_{Y}\right)^{*} A\right|_{Y}$, corresponding to the eigenvalues $\beta_{i}$ $\left(i \in \mathcal{I}_{1}\right)$, by $\eta_{i}$. Furthermore, find the vectors $\left(\eta_{i}\right)_{i \in \mathcal{I}_{0}}$, forming an orthonormal basis in $\operatorname{ker}\left(\left.A\right|_{Y}\right)$. For $i \in \mathcal{I}_{0}$, set $\beta_{i}=0$. Let $\mathcal{I}=\mathcal{I}_{1} \cup \mathcal{I}_{0}$ (we assume that this union is disjoint). Then the family $\left(\eta_{i}\right)_{i \in \mathcal{I}}$ is the canonical basis for $Y$.

For each positive integer $k$, let $M_{k}$ be the smallest value of $i$ s.t. $\beta_{i} \leqslant a^{-k}$. Set $M_{0}=1$. In this notation, $a^{1-k} \geq \beta_{i}>a^{-k}$ iff $M_{k-1} \leqslant i<M_{k}$. As noted above, $\beta_{i} \leqslant \alpha_{2 i}$, hence $M_{k} \leqslant N_{k}$. By our choice of the sequence $\left(N_{k}\right)$,

$$
M_{k}-M_{k-1}<M_{k} \leqslant N_{k} \leqslant N_{k+1}-N_{k} .
$$

Thus, there exists an injective map $\pi: \mathcal{I} \rightarrow \mathbb{N}$ s.t. $\pi\left(\mathcal{I}_{0}\right) \subset\{2 i-1: i \in \mathbb{N}\}$, and $\pi\left(\left[M_{k-1}, M_{k}\right)\right) \subset\left\{2 i: i \in\left[N_{k}, N_{k+1}\right)\right\}$ for each $k \in \mathbb{N}$. For $i \in \mathcal{I}_{0}, \alpha_{\pi(i)}=\beta_{i}=0$, while for $i \in \mathcal{I}_{1}, a \alpha_{\pi(i)}=a^{1-k} \geq \beta_{i}>a^{-k}=\alpha_{\pi(i)}$. Define the operator $T: Y \rightarrow$ $\operatorname{span}\left[e_{\pi(i)}: i \in \mathcal{I}\right] \hookrightarrow X^{d}(\alpha)$, defined by $T \xi_{i}=e_{\pi(i)}$. By (4-1), $T$ is a complete contraction, and $\left\|T^{-1}\right\|_{c b} \leqslant a$.

Remark 5.8 The proof of Theorem 5.6 shows that any subspace of $X^{d}(\alpha)$ is $a$ completely isomorphic to a completely contractively complemented subspace of $X^{d}(\alpha)$. Nevertheless, $X^{d}(\alpha)$ contains subspaces which are not completely complemented. To construct them, find a sequence $\left(\beta_{i}\right)$ such that $1 \geq \beta_{1} \geq \beta_{2} \geq \ldots>0, \lim \beta_{i}=0$, and furthermore, $\sum_{i} \gamma_{i}^{2}=\infty$, where $\gamma_{i}=\alpha_{i} \beta_{i}$. Denote the canonical basis of $X^{d}(\alpha)$ 
by $\left(e_{i}\right)_{i \in \mathbb{N}}$. Then $e_{2 i-1}=\xi_{2 i-1} \oplus 0$, and $e_{2 i}=\xi_{2 i} \oplus \alpha_{i} \xi_{2 i}\left(\left(\xi_{j}\right)\right.$ is an orthonormal basis in $\left.\ell_{2}\right)$. For $k \geq 0$ and $i \in\left[N_{k}, n_{k+1}\right)$, let

$$
f_{i}=\beta_{i} e_{2 i}+\sqrt{1-\beta_{i}^{2}} e_{2 i-1}=\left(\beta_{i} \xi_{2 i}+\sqrt{1-\beta_{i}^{2}} \xi_{2 i-1}\right) \oplus \gamma_{i} \xi_{2 i} .
$$

We show that $Y$ is not completely complemented in $X^{d}(\alpha)$. Indeed, suppose, for the sake of contradiction, that there exists a c.b. projection $P$ from $X^{d}(\alpha)$ onto $Y$. For $\varepsilon \in\{-1,1\}^{\mathbb{N}}$, define an operator $\Lambda_{\varepsilon} \in B\left(X^{d}(\alpha)\right)$ by setting $\lambda_{\varepsilon} e_{j}=\varepsilon_{\lceil j / 2\rceil} e_{j}$. For any such $\varepsilon$, (4-2) implies that $\Lambda_{\varepsilon}$ is a complete isometry. For any $i \in \mathbb{N}$, we have $\Lambda_{\varepsilon} \xi=\varepsilon_{i} \xi$ whenever $\xi \in \operatorname{span}\left[e_{2 i-1}, e_{2 i}\right]$. In particular, $\Lambda_{\varepsilon} f_{i}=\varepsilon_{i} f_{i}$. Let $Q=\operatorname{Ave}_{\varepsilon \in\{-1,1\}^{\mathbb{N}}} \Lambda_{\varepsilon} P \Lambda_{\varepsilon}$. Note that $\operatorname{ran} Q \subset Y$, and $\left.Q\right|_{Y}=I_{Y}$, hence $Q$ is a projection onto $Y$. Furthermore, $\|Q\|_{c b} \leqslant\|P\|_{c b}$.

For each $i$, we have $Q e_{2 i}=a_{i} f_{i}$, and $Q e_{2 i-1}=b_{i} f_{i}$. The equations $Q f_{i}=f_{i}$ and (5-8) yield $a_{i} \beta_{i}+b_{i} \sqrt{1-\beta_{i}^{2}}=1$. Then $\sup _{i} \max \left\{\left|a_{i}\right|,\left|b_{i}\right|\right\} \leqslant\|Q\|$. As $\lim \beta_{i}=0$, there exists $K \in \mathbb{N}$ such that $\left|b_{i}\right|>1 / 2$ for $i>K$. Find $N \in \mathbb{N}$ s.t. $\sum_{i=K+1}^{K+N} \gamma_{i}^{2}>4\|Q\|_{c b}^{2}$ (this is possible, since $\sum_{i} \gamma_{i}^{2}=\infty$ ). Consider $x=\sum_{i=K+1}^{K+N} E_{i 1} \otimes e_{2 i-1} \in M_{N}\left(X^{d}(\alpha)\right.$ ). Then $\|x\|=1$, and therefore,

$$
\begin{aligned}
\|Q\|_{c b} & \geq\left\|\left(I_{M_{N}} \otimes Q\right) x\right\|=\left\|\sum_{i=K+1}^{K+N} E_{i 1} \otimes b_{i} f_{i}\right\| \\
& \geq\left\|\sum_{i=K+1}^{K+N} E_{i 1} \otimes b_{i} \gamma_{i} \xi_{2 i}\right\|_{M_{N}(\mathbf{C})}=\left(\sum_{i=K+1}^{K+N}\left|b_{i}\right|^{2} \gamma_{i}^{2}\right)^{1 / 2} \\
& \geq \frac{\left(\sum_{i=K+1}^{K+N} \gamma_{i}^{2}\right)^{1 / 2}}{2}>\|Q\|_{c b},
\end{aligned}
$$

which is impossible.

Remark 5.9 If any subspace of a Banach space $E$ is complemented, then $E$ is isomorphic to a Hilbert space. This was first proved in [14], see also [10] for sharper asymptotics of the isomorphism constants. A recent preprint [9] exhibits a class of separable Banach spaces $E$, not isomorphic to $\ell_{2}$, such that every subspace of $E$ is isomorphic to a complemented subspace of $E$. It is not known whether $E$ can be constructed in such a way that all of its subspaces have an unconditional basis.

\section{Completely isomorphic classification of subspaces of $X(A)$}

The main goal of this section is to prove Theorem 6.1 and Corollary 6.2 below. Recall that $\mathfrak{C}$ is the set of compact contractions which are not Hilbert-Schmidt. 
Theorem 6.1 If $A \in B\left(\ell_{2}\right)$ belongs to $\mathfrak{C}$, then $(\mathbf{S}(X(A)), \simeq)$ is Borel bireducible to the complete $\mathbf{K}_{\sigma}$ relation.

Together with Corollary 4.5, this theorem immediately implies

Corollary 6.2 If $A \in B\left(\ell_{2}\right)$ belongs to $\mathfrak{C}$, then the relation of complete biembeddability on $\mathbf{S}(X(A))$ is Borel bireducible to the complete $\mathbf{K}_{\sigma}$ relation.

The proof of Theorem 6.1 proceeds in two steps. First, we introduce the space $S_{A}$ of sequences of non-negative generalized integers, with an equivalence relation $\stackrel{*}{\sim}$, and show the latter is Borel bireducible with $(\mathbf{S}(X(A)), \simeq)$. Then we prove that $\left(S_{A}, \stackrel{*}{\sim}\right)$ is, in fact, a complete $\mathbf{K}_{\sigma}$ relation.

Suppose $A \in B\left(\ell_{2}\right)$ is of class $\mathfrak{C}$. By Lemma 3.2, we can assume that $\|A\|<1$, and $A \geq 0$. List the positive eigenvalues of $A$ in the non-increasing order: $1>\|A\|=$ $s_{1}^{o} \geq s_{2}^{o} \geq \ldots>0$. In the terminology of Section $4,\left(s_{i}^{o}\right)_{i \in \mathbb{N}}=\mathbf{D}(A)$. Clearly, $\lim _{i} s_{i}^{o}=0$. Let $\left(\xi_{i}\right)_{i \in \mathbb{N}}$ be the normalized eigenvectors of $A$, corresponding to the eigenvalues $s_{i}^{o}$. We can identify $\operatorname{span}\left[\xi_{i}: i \in \mathbb{N}\right]$ with $\ell_{2}$. Consider the operator $\hat{A}=\operatorname{diag}\left(s_{i}^{o}\right) \oplus 0 \in B\left(\ell_{2} \oplus \ell_{2}\right)$. By Corollary 3.8, $X(A) \simeq X(\hat{A})$. For the rest of this section, we assume that $A=\hat{A}$.

Denote by $\left(\xi_{i}^{\prime}\right)_{i \in \mathbb{N}}$ the canonical orthonormal basis in the second copy of $\ell_{2}$. Then the canonical basis of $X(A)$ is the collection of vectors $e_{i}=\xi_{i} \oplus s_{i}^{o} \xi_{i}$ and $f_{i}=\xi_{i}^{\prime} \oplus 0$. As in (4-1), we have, for $n \times n$ matrices $a_{1}, b_{1}, a_{2}, b_{2}, \ldots$,

$$
\begin{aligned}
& \left\|\sum_{i} a_{i} \otimes e_{i}+\sum_{i} b_{i} \otimes f_{i}\right\|_{M_{n}(X(A))}^{2} \\
& =\max \left\{\left\|\sum_{i} a_{i} a_{i}^{*}+\sum_{i} b_{i} b_{i}^{*}\right\|,\left\|\sum_{i} s_{i}^{o 2} a_{i}^{*} a_{i}\right\|\right\} .
\end{aligned}
$$

For an infinite dimensional $Y \hookrightarrow X(A)$, we let $\left(s_{i}(Y)\right)=\mathbf{D}\left(\left.A\right|_{Y}\right)$. Recalling the definition of $\mathbf{D}$ from Section 4, we see that, if $\operatorname{rank}\left(\left.A\right|_{Y}\right)=\infty$, then $s_{1}(Y) \geq s_{2}(Y) \geq$ $\ldots$ are the positive singular values of $\left.A\right|_{Y}$, listed in the non-increasing order. In the case of $\operatorname{rank}\left(\left.A\right|_{Y}\right)=n<\infty, s_{1}(Y) \geq s_{2}(Y) \geq \ldots \geq s_{n}(Y)$ are the $n$ positive singular values of $\left.A\right|_{Y}$, and $s_{i}(Y)=0$ for $i>n$. In this notation, $s_{k}^{o}=s_{k}(X(A))$. Clearly, $s_{k}(Y) \leqslant s_{k}^{o}$ for any $k$, and any $Y \hookrightarrow X(A)$.

For $k \in \mathbb{N}$, set $\mathbf{n}_{k}(Y)=\sup \left\{\ell \in \mathbb{N}: 2^{1-\ell} \geq s_{k}(Y)\right\}$. The sequence $\mathbf{n}(Y)=\left(\mathbf{n}_{k}(Y)\right)_{k \in \mathbb{N}}$ belongs to $\mathbb{N}_{*}^{\mathbb{N}}$, where $\mathbb{N}_{*}=\mathbb{N} \cup\{\infty\}$ is viewed as the 1-point compactification of $\mathbb{N}$. For $k \in \mathbb{N}$ let $\alpha_{k}=\mathbf{n}_{k}(X(A))$. Define $S_{A}$ as the set of all elements $\beta=\left(\beta_{i}\right)_{i \in \mathbb{N}} \in \mathbb{N}_{*}^{\mathbb{N}}$, 
such that (1) $\beta_{k} \geq \alpha_{k}$ for any $k$, and (2) $\beta_{1} \leqslant \beta_{2} \leqslant \ldots$ Equipping $\mathbb{N}_{*}^{\mathbb{N}}$ with its product topology, we see that $S_{A}$ is closed.

For any infinite dimensional $Y \hookrightarrow X(A)$, the sequence $\left(\mathbf{n}_{k}(Y)\right)_{k \in \mathbb{N}}$ belongs to $S_{A}$. Conversely, for any $\beta \in S_{A}$ there exists $Y \hookrightarrow X(A)$ s.t. $\beta=\mathbf{n}(Y)$. Indeed, suppose $\beta_{k} \in \mathbb{N}_{*}, \beta_{k} \geq \alpha_{k}$ for any $k$, and $\beta_{1} \leqslant \beta_{2} \leqslant \ldots$ Let $g_{i}=\sin \phi_{i} e_{i}+\cos \phi_{i} f_{i}$, with $s_{i}^{o} \sin \phi_{i}=2^{-\beta_{i}}$. We denote $\operatorname{span}\left[g_{i}: i \in \mathbb{N}\right]$ by $\mathbf{Y}(\beta)$, where $\beta=\left(\beta_{i}\right)$. By (6-1), $\mathbf{n}(\mathbf{Y}(\beta))=\beta$.

Define the relation $\stackrel{*}{\sim}$ on $S_{A}$ as follows: $\beta \stackrel{*}{\sim} \gamma$ if there exists $K \in \mathbb{N}$ and $I \subset \mathbb{N}$ s.t. $\left|\beta_{i}-\gamma_{i}\right| \leqslant K$ for any $i \notin I$, and $\sum_{i \in I}\left(4^{-\beta_{i}}+4^{-\gamma_{i}}\right) \leqslant K$. By Corollary $4.5, \beta \stackrel{*}{\sim} \gamma$ iff $\mathbf{Y}(\beta) \simeq \mathbf{Y}(\gamma)$, and conversely, $Y \simeq Z$ iff $\mathbf{n}(Y) \stackrel{*}{\sim} \mathbf{n}(Z)$.

Proposition 6.3 $\mathrm{n}$ and $\mathbf{Y}$ are Borel maps.

This immediately yields:

Corollary $6.4\left(S_{A}, \stackrel{*}{\sim}\right)$ and $(\mathbf{S}(X(A)), \simeq)$ are Borel bireducible to each other.

Proof of Proposition 6.3 First we handle the map Y. We have to show that, for any open set $U \subset X(A),\left\{\beta \in S_{A}: \mathbf{Y}(\beta) \cap U \neq \emptyset\right\}$ is Borel. But $\mathbf{Y}(\beta) \cap U \neq \emptyset$ iff there exist $m \in \mathbb{N}$ and $\lambda_{1}, \ldots, \lambda_{m} \in \mathbb{Q}+i \mathbb{Q}$ s.t. $\sum_{i=1}^{m} \lambda_{i} g_{i} \in U$. Here, the vectors $\left(g_{i}\right)$ come from the definition of $\mathbf{Y}$. Note that, for each $i, g_{i}$ depends solely (and continuously) on $\beta_{i}$. Therefore, for each $m$-tuple $\left(\lambda_{i}\right)_{i=1}^{m}, \sum_{i=1}^{m} \lambda_{i} g_{i} \in U$ is an open condition on $\beta$. Thus, $\left\{\beta \in S_{A}: \mathbf{Y}(\beta) \cap U \neq \emptyset\right\}$ is Borel.

Now consider $\mathbf{n}$. Fix $m, \beta_{m} \in \mathbb{N}$, and show that the set of all $Y \in \mathbf{S}(X(A))$, for which $\mathbf{n}_{m}(Y)>\beta_{m} \in \mathbb{N}$, is Borel. To this end, find a countable set $\mathcal{O}_{m}$ of orthonormal $m$-tuples $\xi=\left(\xi_{1}, \ldots, \xi_{m}\right)$ in $X(A)$, with the property that, for every $\varepsilon>0$, and any orthonormal $m$-tuple $\left(\eta_{1}, \ldots, \eta_{m}\right)$ in $X(A)$, there exists $\xi=\left(\xi_{1}, \ldots, \xi_{m}\right) \in \mathcal{O}_{m}$ s.t. $\left\|\xi_{i}-\eta_{i}\right\|<\varepsilon$ for any $i$. Furthermore, find a set $\Gamma_{m}$ of $m$-tuples $\gamma=\left(\gamma_{1}, \ldots, \gamma_{m}\right) \in \mathbb{C}^{m}$, dense in the unit sphere of $\ell_{2}^{m}$.

The Minimax Principle (see e.g. [2, p. 75]) states that, for an operator $T \in B(H, K)$, we have $s_{m}(T)>b$ iff $H$ has an $m$-dimensional subspace $E$ such that $\|T \eta\|>b$ for any norm one $\eta \in E$. Therefore, $\mathbf{n}_{m}(Y)>\beta_{m} \in \mathbb{N}$ (that is, $s_{m}\left(\left.A\right|_{Y}\right) \geq 2^{-\beta_{m}}$ ) iff for every $\varepsilon>0$ there exists an $m$-tuple of orthonormal vectors $\eta_{1}, \ldots, \eta_{m} \in Y$, s.t. $\left\|\sum_{i=1}^{m} \gamma_{i} A \eta_{i}\right\|>2^{-\beta_{m}}-\varepsilon$ whenever $\sum_{i=1}^{m}\left|\gamma_{i}\right|^{2}=1$. This, in turn, is equivalent to the following statement: for every $r \in \mathbb{N}$, there exists $\left(\xi_{1}, \ldots, \xi_{m}\right) \in \mathcal{O}_{m}$ s.t., for $1 \leqslant i \leqslant m, \mathrm{Ba}\left(\xi_{i}, 1 / r\right) \cap Y \neq \emptyset$ (here, $\mathrm{Ba}(x, c)$ denotes the open ball of radius $c$, with the center at $x$ ), and $\left\|\sum_{i} \gamma^{i} A \xi_{i}\right\|>2^{-\beta_{m}}-1 / r$ for every $\left(\gamma_{i}\right)_{i=1}^{m} \in \Gamma_{m}$. This condition is Borel, hence $\mathbf{n}$ is Borel. 
Lemma $6.5\left(S_{A}, \stackrel{*}{\sim}\right)$ is a $\mathbf{K}_{\sigma}$ relation.

Proof We have to show that the set $F=\left\{(\beta, \gamma) \in S_{A} \times S_{A}: \beta \stackrel{*}{\sim} \gamma\right\}$ is a $\mathbf{K}_{\sigma}$ set, that is, a countable union of compact sets. To this end, define a family of subsets of $S_{A} \times S_{A}$, described below. For $K, n \in \mathbb{N}$ and $I_{n} \subset\{1, \ldots, n\}$, define $F\left(K, n, I_{n}\right)$ as the set of all pairs $(\beta, \gamma)\left(\beta=\left(\beta_{i}\right), \gamma=\left(\gamma_{i}\right)\right)$ with the property that $\left|\beta_{i}-\gamma_{i}\right| \leqslant K$ for $i \in\{1, \ldots, n\} \backslash I_{n}$, and $\sum_{i \in I_{n}}\left(4^{-\beta_{i}}+4^{-\gamma_{i}}\right) \leqslant K$. Let $F(K, n)=\cup_{I_{n} \subset\{1, \ldots, n\}} F\left(K, n, I_{n}\right)$, and $F(K)=\cap_{n} F(K, n)$. It suffices to show that $F=\cup_{K \in \mathbb{N}} F(K)$. Indeed, $F\left(K, n, I_{n}\right)$ is a compact subset of $S_{A} \times S_{A}$, hence so is $F(K, n)$ (as a finite union of compact sets). Furthermore, $F(K)$ is also compact, and $\cup_{K} F(K)$ is $\mathbf{K}_{\sigma}$.

We show first that $F \subset \cup_{K} F(K)$. By definition, $\beta \stackrel{*}{\sim} \gamma$ if there exists $K \in \mathbb{N}$ and $I \subset \mathbb{N}$ s.t. $\left|\beta_{i}-\gamma_{i}\right| \leqslant K$ for any $i \notin I$, and $\sum_{i \in I}\left(4^{-\beta_{i}}+4^{-\gamma_{i}}\right) \leqslant K$. Letting $I_{n}=I \cap\{1, \ldots, n\}$, we see that $(\beta, \gamma) \in F\left(K, n, I_{n}\right)$ for each $n$, hence $(\beta, \gamma) \in F(K)$.

To prove the converse implication, suppose $(\beta, \gamma) \in F(K)$ for some $K$, and show that $\beta \stackrel{*}{\sim} \gamma$. Construct a tree $T \subset\{0,1\}^{\mathbb{N}}$ : for each $n, T \cap\{0,1\}^{n}$ consists of all the sets $I_{n}$ s.t. $\left|\beta_{i}-\gamma_{i}\right| \leqslant K$ for $i \notin I_{n}$, and $\sum_{i \in I_{n}}\left(4^{-\beta_{i}}+4^{-\gamma_{i}}\right) \leqslant K$ (we identify the set of subsets of $\{1, \ldots, n\}$ with $\left.\{0,1\}^{n}\right)$. The set $T$ is indeed a tree: if $I_{n} \in T$, then $I_{n} \cap\{1 \ldots, m\} \in T$ for $m<n$. By assumption, $T$ has arbitrarily long branches. By König's Lemma [11, p. 20], $T$ has an infinite branch, which yields a set $I \subset \mathbb{N}$ s.t. $\left|\beta_{i}-\gamma_{i}\right| \leqslant K$ for $i \notin I$, and $\sum_{i \in I}\left(4^{-\beta_{i}}+4^{-\gamma_{i}}\right) \leqslant K$.

Next consider a space $\Xi=\prod_{k \in \mathbb{N}} \Xi_{k}$, where $\Xi_{k}=\{0, \ldots, k-1\}$, with the equivalence relation $c E_{\mathbf{K}_{\sigma}} b$ iff $\sup _{i}\left|c_{i}-b_{i}\right|<\infty$ (here, $\left.c=\left(c_{i}\right)_{i \in \mathbb{N}}, b=\left(b_{i}\right)_{i \in \mathbb{N}}\right)$. By [24], $E_{\mathbf{K}_{\sigma}}$ is a complete $\mathbf{K}_{\sigma}$ relation, hence, by Lemma 6.5 , it reduces $\left(S_{A}, \stackrel{*}{\sim}\right)$. It remains to prove the converse.

Proposition 6.6 There exists a Borel map $\phi: \Xi \rightarrow S_{A}$ s.t. $\phi(b) \stackrel{*}{\sim} \phi(c)$ iff $c E_{\mathbf{K}_{\sigma}} b$.

Proof Recall that $A$ is a compact contraction which is not Hilbert-Schmidt. Therefore, $\sum_{i} 4^{-\alpha_{i}}=\infty$, and $\lim _{i} \alpha_{i}=\infty$. Thus, there exists a sequence of positive integers $1=p_{0}<q_{1}<p_{2}<q_{2}<\ldots$ s.t. $\sum_{i \in I_{k}} 4^{-\alpha_{i}}>4^{2 k}\left(I_{k}=\left[p_{k}, q_{k}-1\right)\right)$, and $\alpha_{p_{k+1}}>k+q_{k}$. Define $\phi\left(\left(b_{i}\right)\right)=\left(b_{j}^{\prime}\right)$ by setting $b_{j}^{\prime}=\alpha_{j}+b_{k}$ if $j \in I_{k}$, and $b_{j}^{\prime}=\min \left\{\alpha_{j}+k, \alpha_{p_{k+1}}\right\}$ if $q_{k} \leqslant j<p_{k+1}$. Clearly, $\phi$ is a Borel map. Moreover, if $c E_{\mathbf{K}_{\sigma}} b$, then $\phi(b) \stackrel{*}{\sim} \phi(c)$. Suppose, on the contrary, that $b^{\prime} \stackrel{*}{\sim} c^{\prime}$, where $b^{\prime}=\phi(b)$ and $c^{\prime}=\phi(c)$. Then there exists a set $I \subset \mathbb{N}$ and $K \in \mathbb{N}$ s.t. $\left|b_{j}^{\prime}-c_{j}^{\prime}\right| \leqslant K$ for $j \notin I$, and $\sum_{j \in I}\left(4^{-b_{j}^{\prime}}+4^{-c_{j}^{\prime}}\right) \leqslant K$. We shall show that $\left|b_{k}-c_{k}\right| \leqslant K$ for all but finitely many 
$k$ 's. Indeed, otherwise there exist infinitely many $k$ 's s.t. $I_{k} \subset I$ (this follows from the fact that $b_{j}^{\prime}-c_{j}^{\prime}=b_{k}-c_{k}$ for $\left.j \in I_{k}\right)$. But

$$
\sum_{j \in I_{k}}\left(4^{-b_{j}^{\prime}}+4^{-c_{j}^{\prime}}\right) \geq 2 \cdot 4^{-k} \sum_{j \in I_{k}} 4^{-\alpha_{j}^{\prime}}>1
$$

hence

$$
\sum_{j \in I}\left(4^{-b_{j}^{\prime}}+4^{-c_{j}^{\prime}}\right) \geq \sum_{I_{k} \subset I} \sum_{j \in I_{k}}\left(4^{-b_{j}^{\prime}}+4^{-c_{j}^{\prime}}\right)=\infty
$$

a contradiction.

Conclusion of the proof of Theorem 6.1 By Corollary 6.4, $(\mathbf{S}(X(A)), \simeq)$ and $\left(S_{A} \stackrel{*}{\sim}\right)$ are Borel bireducible to each other. By Lemmas 6.5 and 6.6, $\left(S_{A}, \stackrel{*}{\sim}\right)$ is Borel bireducible to a complete $\mathbf{K}_{\sigma}$ relation.

Remark 6.7 For many separable Banach spaces $X$, it is known that the isomorphism relation on $\mathbf{S}(X)$ reduces certain "classical" relations, such as $E_{\mathbf{K}_{\sigma}}$ (see e.g. [1, 4, 5, 6]).

\section{Proofs of Theorems 1.1, 1.2, and 1.3}

Recall that the class $\mathfrak{C}$ consists of all compact contractions, which are not HilbertSchmidt, and the family $\mathfrak{F}$ is the set of all operator spaces $X(A)$, where $A \in B\left(\ell_{2}\right)$ belongs to $\mathfrak{C}$. Clearly, all these spaces are isometric to $\ell_{2}$.

Proof of Theorem 1.1 Suppose $X(A) \in \mathfrak{F}$. By Theorem 6.1 and Corollary 6.2, the relations of complete isomorphism and complete biembeddability on $\mathbf{S}(X(A))$ are complete $\mathbf{K}_{\sigma}$. To show that $\mathfrak{F}$ contains a continuum of spaces, not completely isomorphic to each other, pick $A \in B\left(\ell_{2}\right) \cap \mathfrak{C}$. Consider a space $\Xi=\prod_{k \in \mathbb{N}} \Xi_{k}$, where $\Xi_{k}=\{0, \ldots, k-1\}$, with the equivalence relation $c E_{\mathbf{K}_{\sigma}} b$ iff $\sup _{i}\left|c_{i}-b_{i}\right|<\infty$ (here, $\left.c=\left(c_{i}\right)_{i \in \mathbb{N}}, b=\left(b_{i}\right)_{i \in \mathbb{N}}\right)$. By the results of Section 6, there exists a Borel map $\Phi: \Xi \rightarrow \mathbf{S}(X(A))$, such that $\Phi(b) \simeq \Phi(c)$ iff $b E_{\mathbf{K}_{\sigma}} c$. It remains to find a family $\left(b_{\varepsilon}\right)_{\varepsilon \in\{0,1\}^{\mathbb{N}}} \subset \Xi$, such that $b_{\varepsilon} E_{\mathbf{K}_{\sigma}} b_{\delta}$ iff $\varepsilon=\delta$. To this end, write $\mathbb{N}$ as a disjoint union of infinite sets $I_{k}(k \in \mathbb{N})$. For any $\varepsilon=\left(\varepsilon_{k}\right)_{k=1}^{\infty}$, define

$$
b_{\varepsilon}(i)=\left\{\begin{array}{ll}
0 & i \in I_{k}, \varepsilon(k)=0 \\
i-1 & i \in I_{k}, \varepsilon(k)=1
\end{array} .\right.
$$

Clearly, this family $\left(b_{\varepsilon}\right)$ has the desired properties. 
Proof of Theorem 1.2 Consider $A \in B\left(\ell_{2}\right)$ of class $\mathfrak{C}$. The existence of the canonical basis has been established at the beginning of Section 4 , while its uniqueness follows from Proposition 5.2.

Proof of Theorem 1.3 Combine Theorem 1.2 with Theorem 5.6 and Corollary 5.7.

\section{Isometric classification: proof of Theorem 1.4}

We handle the real case. Begin by introducing a numerical invariant of subspaces of $X=\mathbb{R} \oplus_{1} \ell_{2}$. Denote by $P$ the "natural" projection onto $\mathbb{R}$. For $Y \in \mathbf{S}(X)$, define $c(Y)=\left\|\left.P\right|_{Y}\right\|$.

Lemma 8.1 For $Y \in \mathbf{S}(X)$, there exists $x \in Y$ such that $\|x\|=1$, and $\|P x\|=c(Y)$. Moreover, if this $x$ is written as $x=c(Y) \oplus(1-c(Y)) \xi_{0}$, then $Y=\operatorname{span}\left[x, Y^{\prime}\right]$, where $Y^{\prime}=\left\{0 \oplus \xi: \xi \in\left(Y \cap \ell_{2}\right) \cap \xi_{0}^{\perp}\right\}$.

Proof If $c(Y)=0$, the statement is trivial. Suppose $c(Y)=1$. Then, for every $n \in \mathbb{N}$, there exists $t_{n} \in(1-1 / n, 1]$ and $\xi_{n} \in \ell_{2}$ s.t. $\left\|\xi_{n}\right\|=1$, and $t_{n} \oplus\left(1-t_{n}\right) \xi_{n} \in X$. As $Y$ is closed, $1 \oplus 0 \in Y$.

Next consider $c(Y) \in(0,1)$. Suppose, for the sake of contradiction, that there is no $x$ as in the statement of the lemma. Then for every $n \in \mathbb{N}$ there exist $t_{n} \in(c(Y)-1 / n, c(Y))$, and $\xi_{n} \in \ell_{2}$ s.t. $\left\|\xi_{n}\right\|=1$, and $t_{n} \oplus\left(1-t_{n}\right) \xi_{n} \in Y$. Passing to a subsequence if necessary, we can assume that $\left(\xi_{n}\right)$ is a Cauchy sequence in $\ell_{2}$. Indeed, otherwise there exist $n_{1}<n_{2}<\ldots$ and $\alpha>0$, such that, for any $i,\left\|\xi_{n_{i+1}}-\xi_{n_{i}}\right\|>\alpha$. By the uniform convexity of Hilbert spaces (which follows, for instance, from the parallelogram identity), there exists $\beta>0$ s.t. $\left\|\left(1-t_{n_{i+1}}\right) \xi_{n_{i+1}}+\left(1-t_{n_{i}}\right) \xi_{n_{i}}\right\| / 2<$ $1-c(Y)-\beta$ for any $i$. Define

$$
y_{i}=\frac{t_{n_{i+1}}+t_{n_{i}}}{2} \oplus \frac{\left(1-t_{n_{i+1}}\right) \xi_{n_{i+1}}+\left(1-t_{n_{i}}\right) \xi_{n_{i}}}{2} \in Y .
$$

Then $\left\|y_{i}\right\|<1-\beta$, and $\lim _{i}\left\|P y_{i}\right\|=c(Y)$. Therefore, $\left\|\left.P\right|_{Y}\right\|>c(Y)$, which is impossible.

Thus, the sequence $\left(\xi_{n}\right)$ converges to some $\xi_{0} \in \ell_{2}$. Then $x=c(Y) \oplus(1-c(Y)) \xi_{0}$ is the limit of the sequence $t_{n} \oplus\left(1-t_{n}\right) \xi_{n}$, hence it belongs to $Y$. Clearly, $\|P x\|=c(Y)$, and $Y=\operatorname{span}\left[x, Y \cap \ell_{2}\right]$. Moreover, any $\xi \in Y \cap \ell_{2}$ is orthogonal to $\xi_{0}$. Indeed, 
otherwise there exists $\xi \in Y \cap \ell_{2}$ and $z \in \mathbb{C}$ s.t. $\left\|\xi_{0}+z \xi\right\|<\left\|\xi_{0}\right\|$. Then $x^{\prime}=$ $x+(0 \oplus \xi)=c(Y) \oplus\left(\xi_{0}+z \xi\right)$ belongs to $Y,\left\|x^{\prime}\right\|<1$, and $\left\|P x^{\prime}\right\|=c(Y)$, which is impossible.

For $t \in[0,1]$, define $\phi(t)=t+\sqrt{(1-t)^{2}+1}$. Clearly, $\phi$ is continuous and increasing.

Lemma 8.2 For $Y \in \mathbf{S}(X)$,

$$
\phi(c(Y))=\sup \left\{\liminf _{i}\left\|x+y_{i}\right\|: x, y_{i} \in Y,\|x\|=\left\|y_{i}\right\|=1, y_{i} \stackrel{w}{\rightarrow} 0\right\} .
$$

Moreover, there exist a norm $1 x \in Y$, and a normalized weakly null sequence $\left(y_{i}\right)$ in $Y$, such that $\phi(c(Y))=\left\|x+y_{i}\right\|$ for every $i$.

Proof Assume $c(Y) \in(0,1)$ (only minimal changes are needed to handle $c(Y) \in$ $\{0,1\})$. Write $x=t \oplus(1-t) \xi$ and $y_{i}=t_{i} \oplus\left(1-t_{i}\right) \xi_{i}$. Here, $t, t_{i} \in[0,1], \xi_{i} \in \ell_{2}$, and $\left\|\xi_{i}\right\|=1$. As $y_{i} \rightarrow 0$ weakly, $t_{i} \rightarrow 0$, and $\left\langle\xi, \xi_{i}\right\rangle \rightarrow 0$. Therefore, $\lim _{i}\left\|x+y_{i}\right\|=\phi\left(t_{i}\right)$. Taking the supremum over all $x \in Y$, we prove the desired equality. Furthermore, by Lemma 8.1, $Y=\operatorname{span}\left[x, Y \cap \ell_{2}\right]$, where $x=c(Y) \oplus(1-c(Y)) \xi_{0}, \xi_{0} \in \ell_{2}$ has norm 1 , and $Y \cap \ell_{2}$ is orthogonal to $\xi_{0}$. Let $\left(\xi_{i}\right)$ be an orthonormal basis in $Y \cap \ell_{2}$. Then $\phi(c(Y))=\left\|x+y_{i}\right\|$ for every $i$, and $y_{i} \stackrel{w}{\rightarrow} 0$.

Lemma 8.3 If $Y$ and $Z$ are infinite dimensional subspaces of $X$, and $Y$ is almost isometrically embeddable into $Z$, then $c(Y) \leqslant c(Z)$.

Proof By definition, for every $\lambda \in(1,1.1)$, there exist a subspace $W \hookrightarrow Z$ and a contraction $T: Y \rightarrow W$ with $\left\|T^{-1}\right\|<\lambda$. It suffices to show that

$$
\phi(c(W)) \geq \lambda^{-1} \phi(c(Y))-2(\lambda-1) .
$$

Indeed, then we would conclude

$$
\phi(c(Z)) \geq \phi(c(W)) \geq \lambda^{-1} \phi(c(Y))-2(\lambda-1) .
$$

As the above inequality holds for any $\lambda>1$, we conclude that $\phi(c(Y)) \leqslant \phi(c(Z))$. By the monotonicity of $\phi, c(Y) \leqslant c(Z)$.

By Lemma 8.2, there exists a normalized weakly null sequence $\left(y_{i}\right)$ in $Y$, and a norm one $x \in Y$, such that $\phi(c(Y))=\left\|x+y_{i}\right\|$. In the space $W$, consider the elements $x^{\prime}=T x /\|T x\|$, and $y_{i}^{\prime}=T y_{i} /\left\|T y_{i}\right\|$. Then the sequence $\left(y_{i}^{\prime}\right)$ is weakly null, and

$$
\left\|x^{\prime}+y_{i}^{\prime}\right\| \geq\left\|T\left(x+y_{i}\right)\right\|-\left\|\left(1-\|T x\|^{-1}\right) T x\right\|-\left\|\left(1-\left\|T y_{i}\right\|^{-1}\right) T y_{i}\right\| .
$$


But $\left\|T^{-1}\right\|\left\|T\left(x+y_{i}\right)\right\| \geq\left\|x+y_{i}\right\|$, hence $\left\|T\left(x+y_{i}\right)\right\|>\lambda^{-1} \phi(c(Y))$. Furthermore, $1 \geq\|T x\|>\lambda^{-1}$, hence $\left\|\left(1-\|T x\|^{-1}\right) T x\right\|<\lambda-1$. Similarly, $\left\|\left(1-\left\|T y_{i}\right\|^{-1}\right) T y_{i}\right\|<$ $\lambda-1$. By (8-2), $\left\|x^{\prime}+y_{i}^{\prime}\right\|>\lambda^{-1} \phi(c(Y))-2(\lambda-1)$. Applying Lemma 8.2, we obtain $(8-1)$.

Lemma 8.4 If $Y$ and $Z$ are infinite dimensional subspaces of $X$, and $c(Y)=c(Z)$, then $Y$ is isometric to $Z$.

Proof We consider the case $c(Y)=c(Z) \in(0,1)$ (the extreme cases of $c(Y)=c(Z) \in$ $\{0,1\}$ are handled similarly). By Lemma 8.1, $Y$ contains a norm one $y=c(Y) \oplus \xi_{Y} \in Y$ (note that $\left\|\xi_{Y}\right\|=1-c(Y)$ ), s.t. $Y=\operatorname{span}\left[y, Y \cap \ell_{2}\right]$, and $Y^{\prime}=Y \cap \ell_{2}$ is orthogonal to $\xi_{Y}$. Thus, for any $\xi \in Y^{\prime},\|y+\xi\|=c(Y)+\sqrt{(1-c(Y))^{2}+\|\xi\|^{2}}$. Similarly, $Z=\operatorname{span}\left[z, Z^{\prime}\right]$, and $\|z+\eta\|=c(Z)+\sqrt{(1-c(Z))^{2}+\|\eta\|^{2}}$ for any $\eta \in Z^{\prime}$. As $Y^{\prime}$ and $Z^{\prime}$ are both infinite dimensional separable Hilbert spaces, there exists an isometry $T^{\prime}$ from $Y^{\prime}$ onto $Z^{\prime}$. We complete the proof by defining the isometry $T$ from $Y$ onto $Z$ by setting $T y=z$, and $\left.T\right|_{Y^{\prime}}=T^{\prime}$.

Proof of Theorem 1.4 By Lemmas 8.3 and 8.4, the following statements are equivalent for $Y, Z \in \mathbf{S}(X)$ : (i) $Y$ and $Z$ are isometric, (ii) $d(Y, Z)=1$, (iii) $Y$ and $Z$ are isometrically bi-embeddable, (iv) $Y$ and $Z$ are almost isometrically bi-embeddable, (v) $c(Y)=c(Z)$. Denote that canonical basis for $\ell_{2}$ by $e_{0}, e_{1}, \ldots$, and consider a map

$$
\Phi:[0,1] \rightarrow \mathbf{S}(X): t \rightarrow \operatorname{span}\left[t \oplus(1-t) e_{0}, 0 \oplus e_{1}, 0 \oplus e_{2}, \ldots\right] .
$$

Then $c(\Phi(t))=t$, hence $\Phi\left(t_{1}\right)$ and $\Phi\left(t_{2}\right)$ satisfy any (equivalently, all) of the relations (i) - (iv) iff $t_{1}=t_{2}$. It remains to prove that the maps $\Phi$ and $c$ are Borel.

To handle $\Phi$, consider an open ball $U \subset X$ with the center at $\alpha \oplus \sum_{i=0}^{N} \beta_{i} e_{i}$ and radius $r$. Then $\Phi(t) \cap U \neq \emptyset$ iff there exist $\lambda_{0}, \ldots, \lambda_{N} \in \mathbb{Q}$ s.t.

$$
\left|\alpha-t \lambda_{0}\right|+\left(\left|\beta_{0}-(1-t) \lambda_{0}\right|^{2}+\sum_{i=1}^{N}\left|\beta_{i}-\lambda_{i}\right|^{2}\right)^{1 / 2}<r .
$$

This inequality describes a Borel subset of $[0,1]$. As any open subset of $X$ is a countable union of open balls, the map $\Phi$ is Borel.

To deal with $c$, consider the sets

$$
U_{t}=\left\{s \oplus \xi \in \mathbb{R} \oplus_{1} \ell_{2}:|s|>t,\|\xi\|<\sqrt{1-t^{2}}\right\}
$$

$(t \in[0,1])$. Clearly, $U_{t}$ is an open subset of $X$, and $c(Y)>t$ iff $Y \cap U_{t} \neq \emptyset$.

Remark 8.5 Theorem 1.4 holds not only for $\mathbb{R} \oplus_{1} \ell_{2}$, but also for $\mathbb{R} \oplus_{p} \ell_{2}$, for $1 \leqslant p<2$. 


\section{References}

[1] R. Anisca, The ergodicity of weak Hilbert spaces, Proc. Amer. Math. Soc. 138 (2010), 1405-1413; doi:10.1090/S0002-9939-09-10164-8.

[2] R. Bhatia, Matrix Analysis, Springer Verlag, New York, 1997.

[3] E. Effros and Z.-J. Ruan, Operator Spaces, Oxford University Press, New York, 2000.

[4] V. Ferenczi and E.M. Galego, Some equivalence relations which are Borel reducible to isomorphism between separable Banach spaces, Israel J. Math. 152 (2006), 61-82; doi:10.1007/BF02771976.

[5] V. Ferenczi, A. Louveau, and C. Rosendal, The complexity of classifying Banach spaces up to isomorphism, J. London Math. Soc. 79 (2009), 323-345; doi:10.1112/jlms/jdn068.

[6] V. Ferenczi and C. Rosendal, Complexity and homogeneity in Banach spaces, in Banach Spaces and their Applications in Analysis, ed. by B. Randrianantoanina and N. Randrianantoanina, Walter de Gruyter, Berlin, 2007, 83-110.

[7] S. Gao, S. Jackson, and B. Sari, On the complexity of the uniform homeomorphism relation between separable Banach spaces, Trans. Amer. Math. Soc., to appear.

[8] C. W. Henson, J. Iovino, A. Kechris, and E. Odell, Analysis and Logic, Cambridge University Press, 2002.

[9] W. Johnson and A. Szankowski, Hereditary approximation property, preprint.

[10] N. Kalton, The complemented subspace problem revisited, Studia Math. 188 (2008), 223-257; doi:10.4064/sm188-3-2.

[11] A. Kechris, Classical Descriptive Set Theory, Springer, New York, 1995.

[12] R. Komorowski and N. Tomczak-Jaegermann, Banach spaces without local unconditional structure, Israel J. Math. 89 (1995), 205-226; doi:10.4064/sm149-1-1.

[13] R. Komorowski and N. Tomczak-Jaegermann, Subspaces of $l_{2}(X)$ and $\operatorname{Rad}(X)$ without local unconditional structure, Studia Math. 149 (2002), 1-21; doi:10.1017/S0024610701002174.

[14] Y. Lindenstrauss and L. Tzafriri, On the complemented subspaces problem, Israel J. Math. 9 (1971) 263-269; doi:10.1007/BF02771592.

[15] Y. Lindenstrauss and L. Tzafriri, Classical Banach spaces II, Springer, 1979.

[16] J. Melleray, Computing the complexity of the relation of isometry between separable Banach spaces, MLQ Mathematical Logic Quarterly 53 (2007), 128-131; doi:10.1002/malq.200610032.

[17] T. Oikhberg, Direct sums of operator spaces, J. London Math. Soc. 64 (2001), 144-160; doi:10.1017/S0024610701002174.

[18] T. Oikhberg and H. Rosenthal, Extension properties for the space of compact operators, J. Funct. Anal. 179 (2001), 251-308; doi:10.1006/jfan.2000.3674. 
[19] V. Paulsen, Completely Bounded Maps and Operator Algebras, Cambridge University Press, 2002.

[20] G. Pisier, The Volume of Convex Bodies and Banach Space Geometry, Cambridge University Press, 1989.

[21] G. Pisier, The operator Hilbert space $\mathrm{OH}$, complex interpolation and tensor norms, Mem. Amer. Math. Soc. 122(585):viii+103, 1996.

[22] G. Pisier, An Introduction to the Theory of Operator Spaces, Cambridge University Press, 2003.

[23] G. Pisier and D. Shlyakhtenko, Grothendieck's theorem for operator spaces, Inv. Math. 150 (2002), 185-217; doi:10.1007/s00222-002-0235-X.

[24] C. Rosendal, Cofinal families of Borel equivalence relations and quasiorders, J. Symbolic Logic 70 (2005), 1325-1340; doi:10.2178/js1/1129642127.

[25] N. Tomczak-Jaegermann, A solution of the homogeneous Banach space problem, in Canadian Mathematical Society. 1945-1995, Vol. 3, ed. by J. Carrell and R. Murty, Canadian Math. Soc., Ottawa, ON, 1996, 267-286.

[26] D. Voiculescu, Some results on norm-ideal perturbations of Hilbert space operators, J. Operator Theory 2 (1979), 3-37.

[27] Q. Xu, Embedding of $C_{q}$ and $R_{q}$ into noncommutative $L_{p}$-spaces, $1 \leqslant p<q \leqslant 2$, Math. Ann. 335 (2006), 109-131; doi:10.1007/s00208-005-0732-5.

Timur Oikhberg, Department of Mathematics, The University of California at Irvine, Irvine CA 92697, and Department of Mathematics, University of Illinois at Urbana-Champaign, Urbana, IL 61801

Christian Rosendal, Department of Mathematics, Statistics, and Computer Science (M/C 249), University of Illinois at Chicago Chicago, IL 60607-7045

toikhber@math.uci.edu, rosendal@math.uic.edu

Received: 24 September $2010 \quad$ Revised: 1 March 2011 